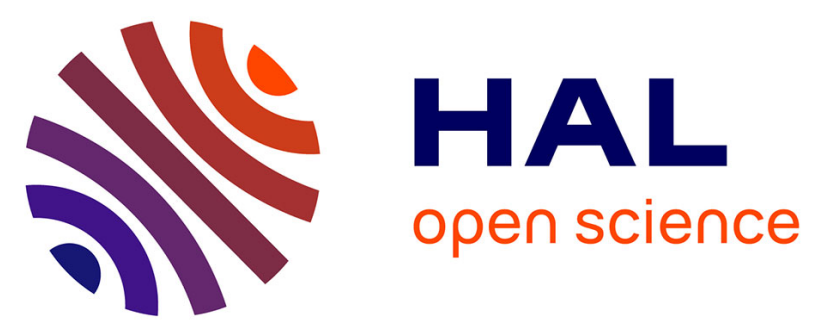

\title{
Micro-mechanical damage of needle puncture on bovine annulus fibrosus fibrils studied using polarization-resolved Second Harmonic Generation(P-SHG) microscopy
}

J.-Y. Wang, J.C. Mansfield, Sophie Brasselet, Claudio Vergari, Judith R. Meakin, Peter C. Winlove

\section{To cite this version:}

J.-Y. Wang, J.C. Mansfield, Sophie Brasselet, Claudio Vergari, Judith R. Meakin, et al.. Micromechanical damage of needle puncture on bovine annulus fibrosus fibrils studied using polarizationresolved Second Harmonic Generation(P-SHG) microscopy. Journal of the mechanical behavior of biomedical materials, 2021, 118, pp.104458. 10.1016/j.jmbbm.2021.104458 . hal-03200723

\section{HAL Id: hal-03200723 \\ https://hal.science/hal-03200723}

Submitted on 16 Apr 2021

HAL is a multi-disciplinary open access archive for the deposit and dissemination of scientific research documents, whether they are published or not. The documents may come from teaching and research institutions in France or abroad, or from public or private research centers.
L'archive ouverte pluridisciplinaire HAL, est destinée au dépôt et à la diffusion de documents scientifiques de niveau recherche, publiés ou non, émanant des établissements d'enseignement et de recherche français ou étrangers, des laboratoires publics ou privés. 


\title{
Micro-mechanical damage of needle puncture on bovine annulus fibrosus fibrils studied using polarization-resolved Second Harmonic Generation (P-SHG) microscopy
}

\author{
J.-Y. Wang ${ }^{\text {a, }}$, J.C. Mansfield ${ }^{\text {a }}$, S. Brasselet ${ }^{\mathrm{b}}$, C. Vergari $^{\mathrm{c}}$, J.R. Meakin ${ }^{\mathrm{a}}$, C.P. Winlove ${ }^{\mathrm{a}}$ \\ ${ }^{\text {a }}$ College of Engineering, Mathematics \& Physical Sciences, Physics Building, Stocker Road, Exeter, EX4 4QL, UK \\ ${ }^{\mathrm{b}}$ Aix Marseille Univ, CNRS, Centrale Marseille, Institut Fresnel, F-13013, Marseille, France \\ ${ }^{\mathrm{c}}$ Arts et Métiers Institute of Technology, Université Sorbonne Paris Nord, IBHGC - Institut de Biomécanique Humaine Georges Charpak, HESAM Université, F-75013, \\ Paris
}

Keywords:

Intervertebral disc degeneration

Needle puncture

Second harmonic generation

Multiphoton microscopy

Disc degeneration

Bovine intervertebral disc

Biomechanics

\begin{abstract}
A B S T R A C T
Needle injection has been widely used in spinal therapeutic or diagnostic processes, such as discography. The use of needles has been suspected in causing mild disc degeneration which can lead to long-term back pain. However, the localised microscopic damage caused by needles has not been well studied. The local progressive damage on a microscopic level caused by needle punctures on the surface of bovine annulus fibrosus was investigated. Four different sizes of needle were used for the puncture and twenty-nine bovine intervertebral discs were studied. Polarization-resolved second harmonic generation and fluorescent microscopy were used to study the local microscopic structural changes in collagen and cell nuclei due to needle damage. Repeated 70 cyclic loadings at $\pm 5 \%$ of axial strain were applied after the needle puncture in order to assess progressive damage caused by the needle. Puncture damage on annulus fibrosus were observed either collagen fibre bundles being pushed aside, being cut through or combination of both with part being lift or pushed in. The progressive damage was found less relevant to the needle size and more progressive damage was only observed using the larger needle. Two distinct populations of collagen, in which one was relatively more organised than the other population, were observed especially after the puncture from skewed distribution of polarization-SHG analysis. Cell shape was found rounder near the puncture site where collagen fibres were damaged.
\end{abstract}

\section{Introduction}

Intervertebral discs (IVD), which lie between vertebral bodies, play a key role in maintaining the flexibility of the spine. Macroscopically, IVD consist of an inner gel-like nucleus pulposus (NP) and a tough outer annulus fibrosus (AF) which has a concentric layer structure with 7-15 lamellae. AF and NP are mostly composed of collagen, proteoglycan, and $60-80 \%$ of water. Any structural damage or mechanical failure can lead to internal disc disruption and result in discogenic pain which has been recognised as one of the main causes of low back pain (Izzo et al., 2015; Ehrlich, 2003). Mechanical injuries can cause strength reduction and delamination of the annulus fibrosus and depressurisation of the nucleus pulposus (Iatridis et al., 2009). Localised mechanical injuries can lead to organ-wide disc damage which results from a disruption in the biochemical balance between cells, extracellular matrix, and disc mechanical stress (Iatridis et al., 2009; Vergroesen et al., 2015). Furthermore, the accumulation of mechanical injuries leads to long-term progressive dysfunction and alteration of biosynthesis in IVD at the macroscopic level (Iatridis et al., 2009). One of the suspected causes of acute injuries is needle puncture which can potentially lead to mild or moderate disc degeneration and low back pain in the long term (Iatridis and Commentary, 2012; Cuellar et al., 2016; Manchikanti et al., 2009; Carragee et al., 2006). Needles are used in spinal diagnostic procedures, such as discography (Bernard, 1990; Guyer et al., 2003), spinal disc repair, and in potential treatments such as electrothermal therapy (Kleinstueck et al., 2001; Cohen et al., 2005; Pollintine et al., 2005; Tsou et al., 2010). The use of needles has been found to accelerate disc degeneration and create progressive degeneration with an increasing number of cell deaths surrounding the puncture site (Masuda et al., 2005; Sobajima et al., 2005; Korecki et al., 2008). Using different

\footnotetext{
* Corresponding author.

E-mail address: j.wang3@exeter.ac.uk (J.-Y. Wang).
} 
sizes of needles can result in different internal damage (Iatridis et al., 2013; Keorochana et al., 2010; Michalet et al., 2010). For example, the use of small needles can cause the reduction of the disc height and nucleus pressurization while using larger needles can disrupt annular function and lower disc stiffness (Michalet et al., 2010; Korecki et al., 2008).

Due to the fact that the damage caused by annular puncture can lead to alterations of biochemical compositions in NP such as water content and collagen, many studies have been done to understand the biomechanics, function, and composition of $\mathrm{AF}$ at the microscopic level (Iatridis et al., 2009). Many microscopic techniques have been used, such as light microscopy (Michalet et al., 2010; Kobielarz et al., 2016), X-ray diffraction (Scott, 1988; Klein and Jukins, 1982; Hickey and Hukins, 1982), electron microscopy (Hickey and Hukins, 1981, 1982; Hayes et al., 2011; Iatridis and Gwynn, 2004; Tavakoli et al., 2017; Nosikova et al., 2012), differential interference contrast optical microscopy (Pezowicz et al., 2005; Schollum et al., 2007, 2018; Brown et al., 2016), and second harmonic generation microscopy (SHG) for collagen (Vergari et al., 2016, 2017a, 2017b; Reiser et al., 2007). While many successes have been made using those techniques, SHG has the advantages of imaging collagen without any staining process (Bueno et al., 2016) and enabling the study of collagen fibre orientation (Brasselet, 2011; Ávila et al., 2015). The working principle of SHG is based on the simultaneous excitation of the sample by two photons of the same frequency, with instantaneous emission of a single photon at twice the frequency (Cox and Kable, 2006). Collagen is a strong source of SHG signal, and collagen fibres can be imaged in tissue without the need of any staining process (Chen et al., 2012). Moreover, the SHG emission from the sample depends on the alignment of the collagen molecules relative to the incident light polarization, where the strongest signal occurs when the molecules are aligned with the incident polarization (Brasselet, 2011; Anceau et al., 2005). This polarization-resolved (or polarization-dependent) SHG has further enabled the analysis on the orientation of collagen and used to study the proportion of disorganised collagen in the tissue (Brasselet, 2011; Ávila et al., 2015). Furthermore, two-photon fluorescent (TPF) signals are often used to image cells while simultaneously acquiring the SHG signal from collagen (Mansfield et al., 2008, 2009; Matcher, 2015; Reed et al., 2019; Bowles et al., 2010; Dittmar, 2008). TPF is also a multiphoton imaging technique and is based on the absorption of two photons followed by the emission of a single photon at a shifted frequency with respect to SHG. As a result of the combined system, SHG-TPF microscopy can provide comprehensive tissue information on the extracellular matrix and cells, and therefore stands out as a very powerful investigative tool (Lacomb et al., 2008; Campagnola, 2011).

This work aimed to investigate the short-term progressive damage caused by needle puncture to the AF, and quantify how this damage is affected by needle size. It is still unclear how a localised needle puncture can induce organ-wide disc degeneration clinically. Based on our previous study, punctures produced no significant changes in elastic properties of AF strips at a relatively large distance from the puncture site (4-6 times the needle diameter) even using different size of needles (Vergari et al., 2017a). In the current study, polarization-resolved SHG combined with TPF microscopy was exploited to study collagen fibres and cell nuclei in the outer AF surrounding the punctures to investigate local microscopic damage. The progressive mechanical damage after needle puncture was also investigated by applying cyclic loadings axially to imitate the effects of spinal motion.

\section{Materials and methods}

\subsection{Sample preparation, cell staining}

Twenty-nine spinal motion segments (an intervertebral disc and two vertebral bodies) from ten cow tails were obtained from a local abattoir. Tails were frozen at $-20^{\circ} \mathrm{C}$ on the day of death and thawed a few hours before testing at room temperature, with maximal frozen time of less than two months. Each motion segment was carefully dissected to remove muscles and ligaments. Intervertebral disc height and diameter were measured using callipers. A thin layer of petroleum jelly was applied on the surface of the outer annulus fibrosus and endplates except for an area of less than $5 \times 5 \mathrm{~mm}^{2}$ on outer AF, which was left exposed for imaging.

Prior to imaging, a few drops of propidium iodide solution with concentration $1 \mathrm{mg} / \mathrm{mL}$ in phosphate-buffered saline (Propidium Iodide $10 \mathrm{mg}$, Sigma-Aldrich, UK) was applied on the $5 \times 5 \mathrm{~mm}^{2}$ imaging area to stain the cell nuclei in the outer annulus fibrosus. Samples were then covered with cling film and aluminium foil and left in a $37{ }^{\circ} \mathrm{C}$ incubator for $40 \mathrm{~min}$. After the staining, disc samples were rinsed with PBS to remove excess propidium iodide on the sample surface.

\subsubsection{Tensile loading and needle puncture}

A custom-built micro-straining rig was utilized to test the samples as described in our previous study (Vergari et al., 2017a). In brief, the rig is motorized with a drive and the driving distance is set at $17 \mu \mathrm{m}$ per step. The sample was mounted in the rig (Fig. 1(a)), and it was loaded with the protocol shown in Fig. 1(b). Each sample was strained between $-5 \%$ and $+5 \%$ in $1 \%$ steps at $5 \mathrm{~mm} / \mathrm{min}$ (corresponding to $0.65 \%$ strain/s). Each cycle lasted between $18.4 \mathrm{~s}$ and $30.8 \mathrm{~s}$ (with frequency range between 0.033 and $0.054 \mathrm{~Hz}$ ) corresponding to the disc thickness 7.65 $\mathrm{mm}-12.85 \mathrm{~mm}$. Polarized SHG images were acquired after each $1 \%$ strain step, with the method described below, while the strain was kept constant. The sample was then unloaded, punctured with the needle radially into the nucleus pulposus using the protocol described below, and then loaded again between $-5 \%$ and $+5 \%$ with an image acquired at each $1 \%$ loading step. Finally, 70 cyclic loadings were performed, with one SHG image acquired every 10 cycles. Images from $1 \%$ loading steps were not included in this study and will leave for future work in comparison with our previous studies in Vergari et al. (2016 and 2017).

Four different needles (Fig. 1(c)) were used to puncture the discs: two hypodermic needles with diameters of $0.5 \mathrm{~mm}(25 \mathrm{G})$ and $0.8 \mathrm{~mm}$ (21G) (Birmingham Gauge) (Yentis et al., 2013), and two spinal needles with diameters of $0.7 \mathrm{~mm}(22 \mathrm{G})$ and $0.9 \mathrm{~mm}(20 \mathrm{G})$ (BD ${ }^{\mathrm{TM}}$ Quinke Spinal Needles, UK). The tips of hypodermic needles have a smaller bevel angle $\left(30^{\circ}\right)$ than the tips of the spinal needles $\left(45^{\circ}\right)$. Punctures were approximately located in the centre of each discs. The exact circumferential position of the puncture sites were not recorded in this study and the locations could vary. The openings of needle tips were randomly aligned. The puncture scheme was shown in Fig. 1(d) and (e) where the needle punctured radially and applied loading axially. Each needle type was applied to five to six discs, and an additional eight discs without puncture formed a control group. Each disc sample was rinsed once using a few drops of PBS solution on the surface of the $5 \times 5 \mathrm{~mm}^{2}$ imaging area prior to the puncture. The acquisition time for each images was approximately $2 \mathrm{~min}$ and $34 \mathrm{~s}$. For control group, the whole protocol lasted within $2.5 \mathrm{~h}$. For needle groups, the whole protocol lasted less than $3.5 \mathrm{~h}$ with less than $2.5 \mathrm{~h}$ just before the puncture and after a few drops of PBS being applied on the imaging area.

The effect of dehydration could affect the collagen behaviour. Although the outer annulus and endplates were covered with petroleum jelly except an $5 \times 5 \mathrm{~m}^{2}$ area, while two vertevrate were also exposed to the air. Force-distance curves were recorded (as force-distance) and compared the changes between the initial and last cyclic loadings within total time period of $2.5 \mathrm{~h}$ using the sample protocols mentioned above.

\subsection{Multiphoton imaging}

The second-harmonic generation imaging system was set up as previously described (Vergari et al., 2016). Both SHG and TPF signals were collected to visualise collagen and cell nuclei, respectively. An $810 \mathrm{~nm}$ mode-locked femto-second Ti:Sapphire laser (Mira 900-D, Coherent Inc.) with a repetition rate of $76 \mathrm{MHz}$ and a pulse width of $100 \mathrm{fs}$ 
(a)

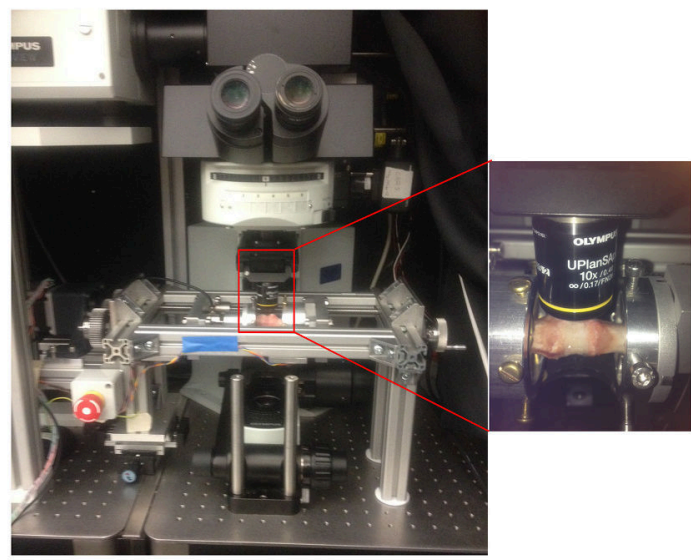

(b)

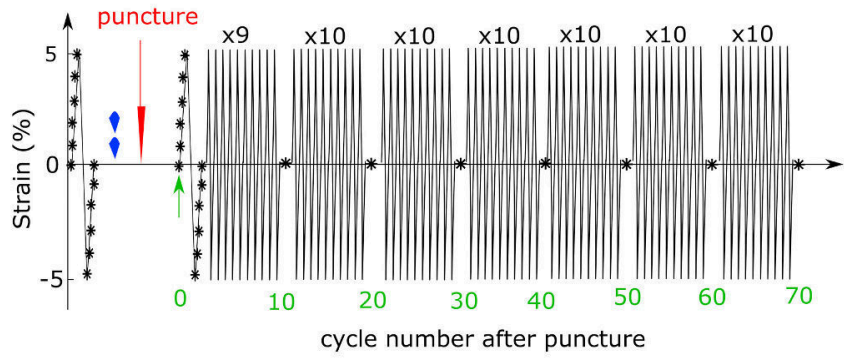

(c)

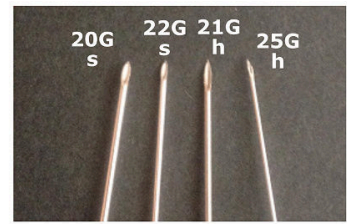

(d)

Imaging from the top

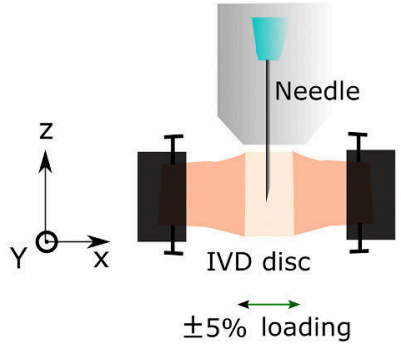

(e)

Imaging area; puncture site

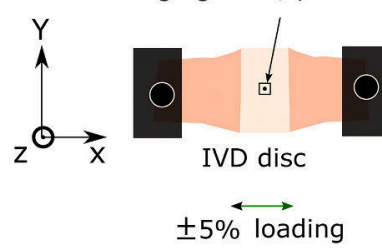

Fig. 1. (a) A bovine tail motion segment was fitted onto a custom-built rig and placed under a microscope. (b) Protocol for puncture and cyclic loadings axially. Images (marked by *) were taken every $1 \%$ step of axial strain during loading and then every 10 cycles during cyclic loadings. A few drops of PBS were applied for rinsing the imaging area on AF surface (marked by blue drops) after initial loading and prior to the puncture. (c) Four different needles used in this study: two hypodermic needles with diameter $0.5 \mathrm{~mm}(25 \mathrm{G})$ and $0.8 \mathrm{~mm}(21 \mathrm{G})$; two Quinke spinal needles with diameter $0.7 \mathrm{~mm}(22 \mathrm{G})$ and $0.9 \mathrm{~mm}(20 \mathrm{G})$. (d) Illustration of needle puncture along radial axis (z) while loading was applied axially along $\mathrm{x}$. (e) Imaging area (puncture site) on $\mathrm{x}-\mathrm{y}$ plane.

pumped by a $532 \mathrm{~nm}$ solid-state laser (Verdi V10, Coherent Inc.) was used as an illumination source. A polarization half-wave plate (WPH05M-488, Thorlabs, Newton, NJ) was mounted on a motorized rotation stage (PR50CC, Newport, Irvine, CA) and the polarization angle was rotated from $0^{\circ}$ to $162^{\circ}$ relative to the laser polarization at $18^{\circ}$ steps which generated a set of ten SHG and TPF images. A confocal microscope (Olympus Fluoview BX51, OLYMPUS Corp., USA) fitted with a 10x/0.4NA air objective (UPlanSApo, OLYMPUS Corp.le, USA) was used to collect both SHG and TPF signal in the backwards direction. The air objectives was used due to it can give the largest imaging areas or field of view, compared to other water or oil objectives designed for magnification higher than 20x. The TPF and SHG signals were separated using a dichroic beam splitter (Semrock Di02-R405), and detected using PMTs (R3896 Hamamatsu Japan). At the SHG PMT the following filters were used (blue green glass filter CG-BG-39 CVI laser and $405 \mathrm{~nm}$ band pass filter, FF01-405/10-25 Semrock) and at the TPF PMT a $655 \mathrm{~nm}$ band pass filter (FF01-655/40-25 Semrock, IDEX Health \& Science, LLC, USA) was used. Disc samples were fitted on the rig and placed under the objective, as shown in Fig. 1(a). Images were mainly collected at two different sizes: $800 \times 600$ pixels $(1600 \mu \mathrm{m} \times 1200 \mu \mathrm{m}$, for needle group $22 \mathrm{G}, 21 \mathrm{G}, 20 \mathrm{G}$ ) with each pixel $2 \mu \mathrm{m} \times 2 \mu \mathrm{m}$ or $512 \times 512$ pixels (1413 $\mu \mathrm{m} \times 1413 \mu \mathrm{m}$, control group and needle group 25G, 21G) with each pixel size $2.76 \mu \mathrm{m} \times 2.76 \mu \mathrm{m}$ due to puncture shape being more rounded for the $25 \mathrm{G}$ needle group and more elongated for three samples in the $21 \mathrm{G}$ needle group. The damage on one sample in $20 \mathrm{G}$ ( $0.9 \mathrm{~mm}$ diameter) created a much larger size of puncture and thus the image was taken as $1024 \times 1024$ pixels $(1.86 \mu \mathrm{m} \times 1.86 \mu \mathrm{m})$.

\subsection{Polarization-resolved $S H G$ and image analysis}

The SHG signal results from how the incident light interacts with non-centrosymmetric assemblies of molecules, in which each molecule can be seen as a dipole radiating at the exactly twice the frequency of the incident light. The polarization dependence of the SHG intensity can be deduced from a theoretical distribution of nonlinear induced dipoles, supposing a coherent superposition of all dipoles in the focal spot of the excitation objective (Brasselet, 2011). Under the dipolar approximation that holds within the size of the diffraction limit size, each nonlinear contribution can be summed up coherently (Brasselet, 2011). SHG from a single molecule (or peptide bond in the case of collagen) is the result of the radiation from the induced individual dipole being linearly polarized along an angle $\alpha$ with respect to $X$ in the sample plane $(X, Y)$ (Brasselet, 2011). At the ensemble level, these dipoles radiate coherently within the focal volume, which results in a macroscopic nonlinear induced dipole $\boldsymbol{P}_{S H G}$ (Mansfield et al., 2019). The measured SHG intensity $\boldsymbol{I}_{S H G}$ can be deduced from the resulting radiated field which leads to a paraxial approximation $\boldsymbol{I}_{S H G} \propto\left|\boldsymbol{P}_{S H G}\right|^{2} \propto|\boldsymbol{E}(\boldsymbol{\alpha})|^{4}$, where $\boldsymbol{E}(\alpha)$ is the incident radiation field. SHG intensity can thus be decomposed in contributions of different harmonics (Mansfield et al., 2019)

$I_{S H G}(\alpha) \propto a_{0}+a_{2} \cos 2 \alpha+b_{2} \sin 2 \alpha+a_{4} \cos 4 \alpha+b_{4} \sin 4 \alpha$

The coefficients can be grouped into amplitude $\left(I_{2}, I_{4}\right)$ and phase coefficients $\left(\phi_{2}, \phi_{4}\right)$ of different orders (2nd or 4 th) of symmetry:

$I_{S H G}(\alpha) \propto a_{0}+I_{2} \cos 2\left(\alpha-\phi_{2}\right)+I_{4} \cos 4\left(\alpha-\phi_{4}\right)$

With:

$$
\begin{aligned}
I_{2}=\frac{\sqrt{a_{2}^{2}+b_{2}^{2}}}{a_{0}}, I_{4} & =\frac{\sqrt{a_{4}^{2}+b_{4}^{2}}}{a_{0}}, \phi_{2}=0.5 \tan ^{-1}\left(\frac{b_{2}}{a_{2}}\right), \phi_{4} \\
& =0.25 \tan ^{-1}\left(\frac{b_{4}}{a_{4}}\right)
\end{aligned}
$$


These parameters are related to how the nonlinear induced dipoles from collagen molecules distribute in the sample plane, which is directly related to the isotropic organization of collagen fibre (Duboisset et al., 2012; Mansfield et al., 2019; Tilbury et al., 2014; Ait-Belkacem et al., 2012; Deniset-Besseau et al., 2009). The second-order parameters $\left(I_{2}\right.$, $\phi_{2}$ ) represent the magnitude and orientation of the anisotropic contribution to the polarization response. i.e. the degree of the order and the overall alignment of the collagen molecules. Higher values of $I_{2}$ represent more anisotropic distribution of collagen molecules, i.e. more orientally ordered or more tightly aligned collagen molecules and thus more organised collagen fibrils within one focal spot (Duboisset et al., 2012; Mansfield et al., 2019; Tilbury et al., 2014; Aitt-Belkacem et al., 2012; Deniset-Besseau et al., 2009). The fourth-order parameters $\left(I_{4}, \phi_{4}\right)$, which are not exploited in this work, are the magnitude and orientation signatures of a more refined higher-order dependence which provides information about the shape of the distribution (Gaussian, cone, or cone surface) and identifies the effect of birefringence (Duboisset et al., 2012; Mansfield et al., 2019).

In this work, we focused on the degree of order of the collagen molecules, to identify how the collagen fibril organization affected by needle puncture. By calculating the coefficients $\left(a_{0}, a_{2}, b_{2}\right), I_{2}$ value was derived from this generic model at each pixel. A custom programme was written using commercial software (MATLAB, 2017b; Mathwork, USA) to calculate the parameters and $I_{2}$ was normalised by the total number of pixels. $I_{2}$ distribution was plotted as histograms and fitted by two Gaussian distribution curves to provide two mean values $\mu_{1}$ and $\mu_{2}$. This is due to the apparent non-normal distributions observed, for which a biGaussian function corresponds to the lowest order fitting basis (Buys and De Clerk, 1972). 200 intervals were set to $I_{2}$ distribution histograms from 0 to 0.8 with 0.0004 in each interval. 900 iterations were set to obtain the best fitting curves using the MATLAB. The mean $I_{2}, \mu_{1}$ and $\mu_{2}$ values, and their changing rates with cyclic loadings were calculated. The other two parameters, the standard deviations $\left(\sigma_{1}, \sigma_{2}\right)$ and the mixing proportions $\left(P_{1}, P_{2}\right)$, were listed in Supported Material SI2 for all the samples at cycle 0,30 to cycle 70 .

\subsection{Statistics}

$I_{2}, \mu_{1}$ and $\mu_{2}$ values calculated from polarized-SHG results were taken average from whole image, and separated in different needle groups. The mean \pm standard deviation were plotted for each needle group. The changing rates versus cyclic loadings of $I_{2}, \mu_{1}$ and $\mu_{2}$ values were taken as the slopes of the fitted linear curve. The mean \pm standard deviation of the changing rates are plotted for each needle group. The Kruskal-Wallis tests were performed to compare the $I_{2}, \mu_{1}, \mu_{2}$ values and their slopes against cycles between needle groups at $5 \%$ significance level. $I_{2}, \mu_{1}, \mu_{2}$ values and their slopes were also normalised by their values from cycle 0 , in order to eliminate the contribution of damage from dissections. Pearson correlation coefficients weer also calculated to obtain the linear correlations between needle size and the averaged $I_{2}, \mu_{1}, \mu_{2}$ values. The Kruskal-Wallis tests were also performed to compare two mixing proportions $P_{1}$ and $P_{2}$ (both non-normalised and normalised values by cycle 0) against needle sizes at $5 \%$ significance level and Pearson correlation coefficients were calculated to obtain their linear correlations with the cycle number. Percent overlap of $I_{2}$ histogram was calculated against cycle number and Kruskal-Wallis tests were also performed between groups. Pearson correlation coefficients were calculated to obtain the linear correlation between percent overlap and cycle number.

Change in puncture length and width were compared after the puncture (cycle 0) and after the cyclic loadings (cycle 70). This is to compare the effect of applied loadings on puncture size changes. The length and width were chosen as along the perpendicular or in parallel to the loading direction, respectively. The change of percentage in length $(\Delta L)$ was defined in this study as the maximum length difference between cycle $0(L O)$ and cycle 70 ( $(L 7)$ devided by $L O$, ie, $\Delta L=(L 7-L O) /$ $L O$. The change of percentage in width $(\Delta w)$ was defined as the maximum width found in cycle $0(w 0)$ and the change the width in the same position after cycle $70(w 7)$ and devided by $w 7$, ie, $\Delta w=(w 7-w 0) /$ $w 0$. The mean and standard deviation for each needle group were calculated. The Kruskal-Wallis tests were performed among and between each needle group, where the change in length and width were compared between each group. Pearson correlation coefficients were calculated to obtain the correlation between needle sizes and percentage changes in puncture sizes (width and length).

Cell length $(l)$ to diameter $(r)$ ratios were calculated and compared between an unpunctured and a punctured sample near the puncture site from the SHG images. $l$ to $r$ ratio of the cells from the whole images were taken average and the standard deviation were calculated.

\section{Results}

\subsection{Needle puncture damage on outer annulus fibrosus}

Fig. 2 shows the SHG images from forty samples (with eight samples in each group, including the control group and four needle groups), at the puncture site immediately after the puncture and before cyclic loading. The shape and size of the puncture varied greatly even using the same needle; with puncture dimensions ranging between 200 and 700 $\mu \mathrm{m}$. Overall, damage from the puncture included both cutting and splitting of collagen bundles, where collagen fibres were cut through or pushed aside, with bundles being torn apart. From the observation, the shape of the puncture can be classified into elongated cracks along the fibre orientation (25G-2, 21G-3), round holes (25G-4, 22G-2 21G-1), or a combination of both. Furthermore, some puctures can be seen with part of the surrounding fibres being pushed into (22G-1,3:6, 21 G-2,4:6, 20G$2,4: 5,7: 8)$ or lifted (21G-2:5) from the sample surface.

Comparing the damage before and after cyclic loadings, i.e. cycle 0 and cycle 70 , changes in shape and size of the puncture were observed as shown in the representative samples in Fig. 3(a). Those representative samples have apparent puncture damage of both tearing and cutting. Among them, some punctures expanded over time (25G-6, 20G-8) while some appeared to shrink (22G-7, 21G-5). Changes in lengths $(L)$ and widths $(w)$ of the puncture before and after 70 cyclic loadings (cycle 0 : $L 0, w 0$, cycle 70: $L 7, w 7$ ) from four needle groups were plotted in Fig. 3 (b-c), respectively. A few samples were not taken into account (G25-6, G22-1, G22-4, G21-1, G21-4, G20-3, G20-5) due to part of the tissue were lifted up towards the end of 70 cycles rather than shrinking or expanding in puncture size. The results from the Kruskal-Wallis tests showed that no significant difference was found between $\Delta w$ and $\Delta L$ within each needle group where $p$-values $=0.564(25 \mathrm{G}), 0.602(22 \mathrm{G})$, 0.513 (21G), 0.756 (20G). Between needle groups, there was no significant difference in change of length $(\Delta L)$ where the $p$ value $p(\Delta L)=$ 0.116 , and width $(\Delta w)$ where $p(\Delta w)=0.402$ within $5 \%$ confidence. Negative correlation was found between needle size and $\Delta L$ with correlation coefficient -0.539 while positive correlation was found between needle size and $\Delta w$ with correlation coefficient 0.648 . For all those 24 samples, the average length change $\Delta L$ was $-13.62 \pm 16.11 \%$ (Mean \pm Std Dev) and width change $\Delta w$ was $-16.60 \pm 15.21 \%$ (Mean \pm Std Dev). There were 1 samples out of 24 expanding in width, and 4 samples expanding in length. These indicated that the majority of the punctures tend to shrink and the applied cyclic loadings caused 3\% less shrinkage in length which is perpendicular to the loading compared to in width. SHG images from all the samples taken at cycle 0 , cycle 30, and cycle 70 are provided in supported material SI1.

Dehydrations can affect the mechanical properties of collagen. The force-distance curves (cycle 1, 2-10, 61-70) from a representative sample in control, needle groups (25G and 20G) were plotted in Fig. 3 (d-f). Some intervals appeared in cycle 1 with relaxations due to the imaging at $1 \%$ steps. Comparing cycle 2 and cycle 70 , forces changed between $-10.4 \%$ and $-16.2 \%$ from Fig. 3(d-f). From all samples, on average, forces changed $-10.2 \pm 3.14 \%$ for control group, and changed $-14.5 \pm 3.5 \%,-17.2 \pm 2.2 \%,-14.4 \pm 2.5 \%,-18.9 \pm 3.4 \%$ for needle 
1
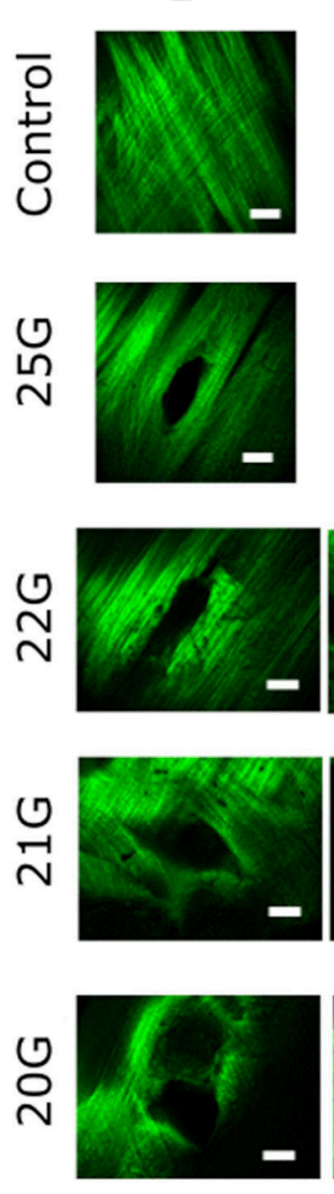

2
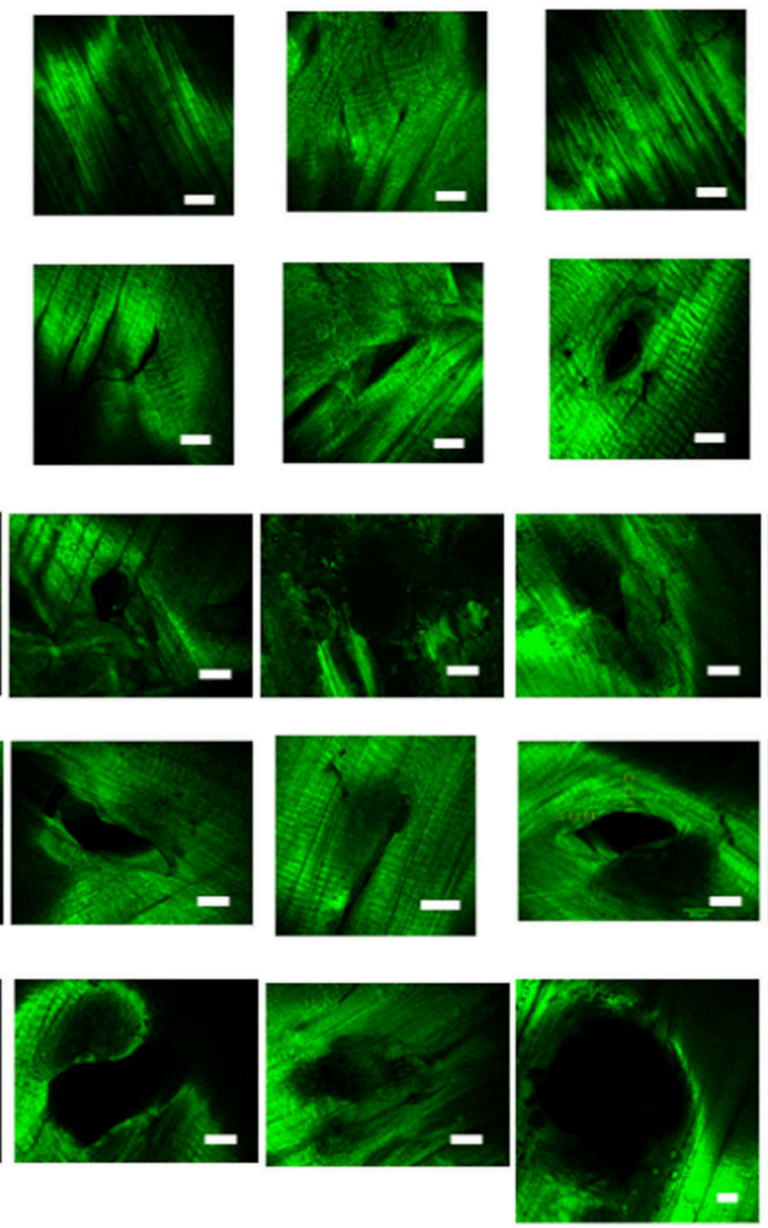

5
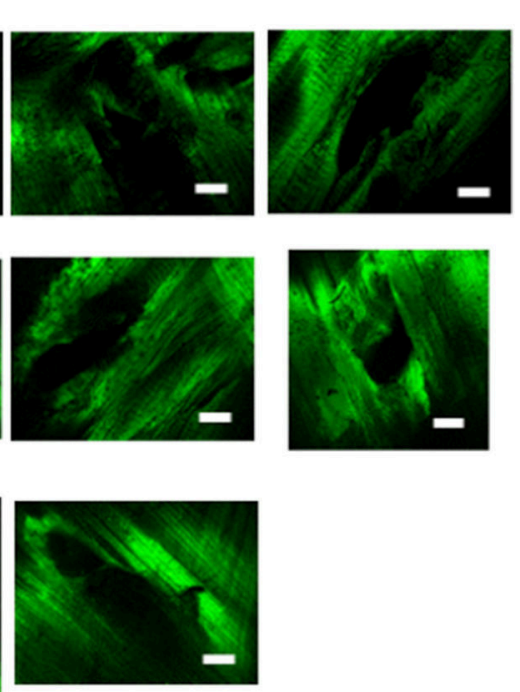

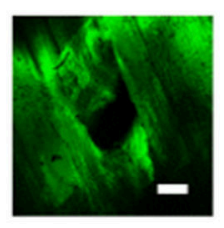

6
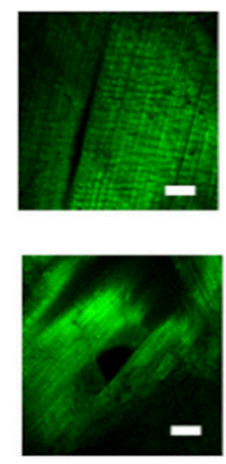

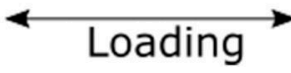

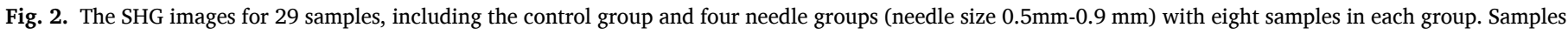
are numbered 1 to 8 in the order of increasing progressive damage. Scalebars represent $200 \mu \mathrm{m}$. The arrow represents the direction of loading.

groups 25G-20G, respectively.

\subsection{Polarized-SHG image analysis}

Fig. 4 shows $I_{2}$ mapping from the same representative samples shown in Fig. 3 at cycle 0,30 and $70 . I_{2}$ value ranges from 0 to 0.8 where the higher $I_{2}$ represents more ordered collagen molecules present in each pixel, i.e. more collagen molecules are aligned to the same orientation. Comparing the needle group with the control group, the change in $I_{2}$ over cyclic loading, indicate that collagen fibril organization decreased, especially in the regions surrounding the puncture, while little change was observed in the control group, even with surface dissected and exposed to the air.

Fig. 5 shows the histogram of $I_{2}$ from the images in Fig. 4, after cycle 0,30 and 70, normalised by the total number of pixels. Shifts in overall $I_{2}$ values over cyclic loadings can be observed from the needle groups compared to the control group. For each $I_{2}$ distribution, non-normal distributions were observed and two Gaussian curves were fitted as shown in the inset of Fig. 5(d and e: Cyc 70) with two mean values $\mu_{1}$ and $\mu_{2}$ representing a population of more organised collagen and less organised collagen respectively. The fitted parameters of those Gaussian distributions, including mean values $\left(\mu_{1}, \mu_{2}\right)$ standard deviations $\left(\sigma_{1}, \sigma_{2}\right)$ and mixing proportions $\left(P_{1}, P_{2}\right)$ were listed in Supported Material SI2. 26 out of 29 samples where $\mu_{1}$ decreased with increasing cycle number while no obvious trends for the other two parameters in both distributions. Sample 20G-1 had very high $\mu_{2}$ from cycle 30-70 (>1) but with very low proportion $P_{2}(<0.1 \%)$ which were assumed as single perfect normal distributions with $P_{2}, \mu_{2}, \sigma_{2}$ being disregarded.

The averaged percent overlap of $I_{2}$ distribution compared to cycle 0 , as illustrated in wavy area in Fig. 5(c), from five groups were plotted in Fig. 5(f) against cycle numbers. Decreases in percent overlap were observed in all groups ranging from $15 \%$ to $25 \%$ on average after 70 cyclic loadings. Kruskal-Wallis tests were performed compared control and four needle groups where $p=0.361(>5 \%)$, which indicated no significant difference found between groups, ie, different needle sizes. Decreasing trends have been observed in all five groups against cycles, and the correlation coefficients for different group against cycle number were -0.9073 (Control), -0.8427 (25G), -0.970 (22G), -0.788 (21G) and -0.9165 (20G) which indicated strong correlations between the loss of percentage overlap against increasing cycles.

The mean $I_{2}, \mu_{1}$ and $\mu_{2}$ values from each group are shown in Fig. 6 (a-c) and their normalised values are shown in Fig. 6(d-f). All the values were taken as averages from all the samples over 70 cycles, i.e., the mean values from all samples with eight data points for each sample. Error bars represent the standard deviation from eight samples. For nonnormalised results, on average larger needles caused more damage compared to the smaller needles, and there is an overall decrease of $I_{2}$ values with increase of needle size while variations occurred in needle 
(a)

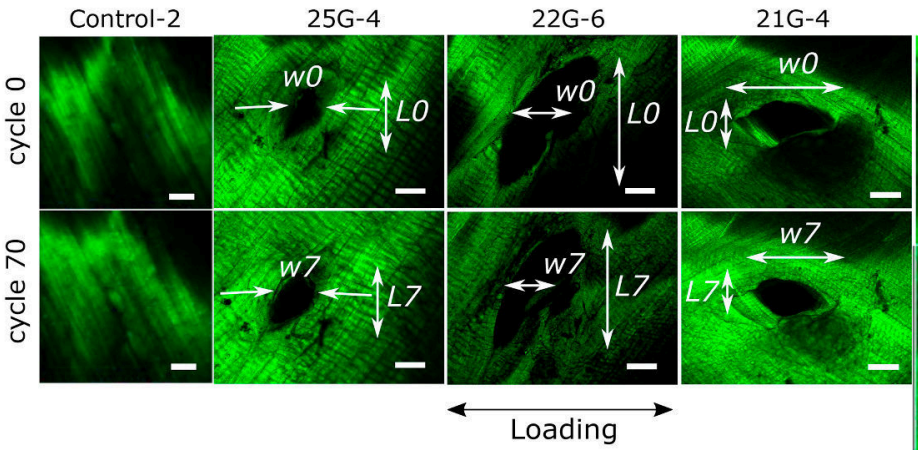

(c) (e) $25 G$ (d) Control

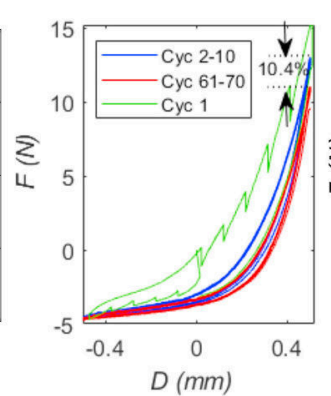

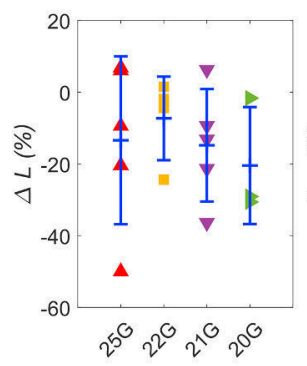
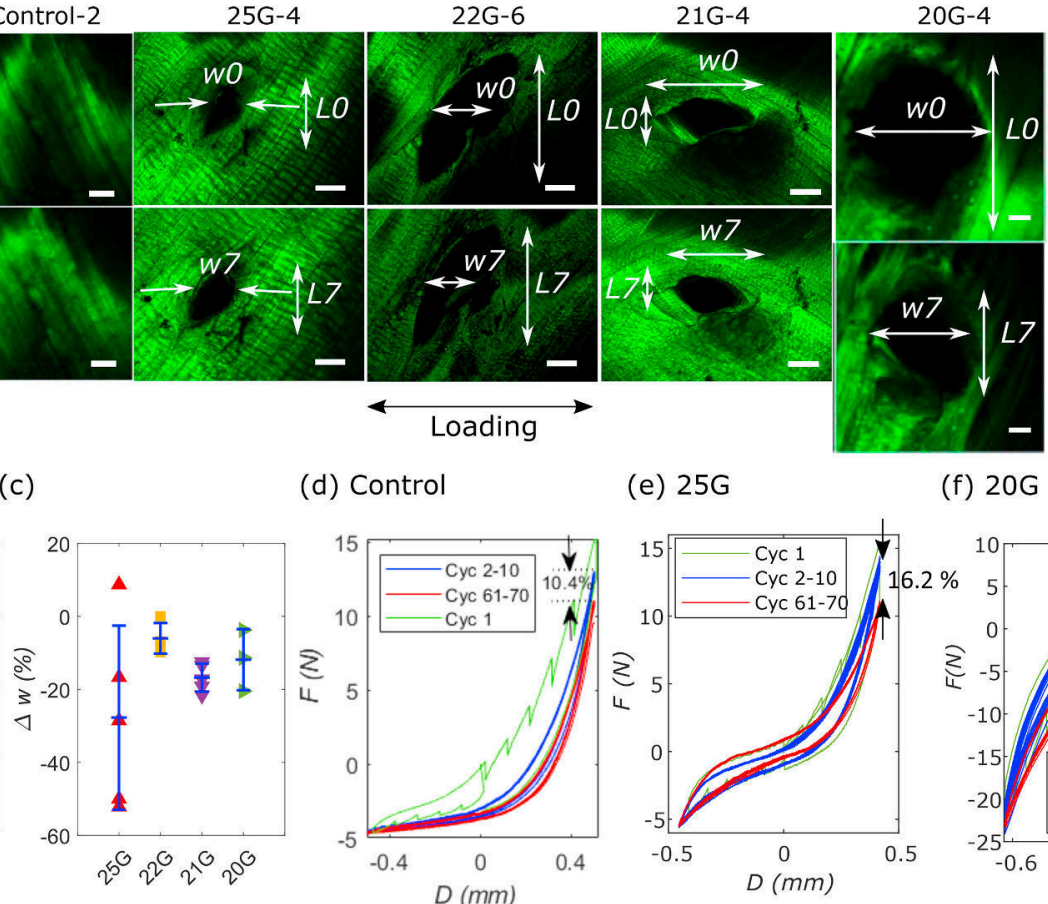

(f) $20 \mathrm{G}$

Fig. 3. (a) SHG collagen fibre images in outer annulus fibrosus from representative samples in each group with obvious damage of cutting and tearing (specimen number referred to Fig. 2). Images taken from before and after 70 cyclic loadings (marked as cycle 0 on top row and cycle 70 on bottom row) are shown here to compare the effect from cyclic loading. All scale bars represent $200 \mu \mathrm{m}$. The arrow represents the direction of loading. The maximum width $(w)$ and length $(L)$ of the puncture before and after the cyclic loading $(w 0, L O, w 7, L 7)$ were marked in each needle groups, respectively, (b) change of maximum puncture length $\Delta L$ and (c) change of maximum puncture width $\Delta w$. $(\mathrm{d}-\mathrm{f})$ The force $(F)$ versus strain $(D)$ cuves for three representative samples from control, $25 \mathrm{G}$ and $20 \mathrm{G}$ needle group on cycle 1 (Cyc1) with intervals for imaging every $1 \%$ step, cycles $2-10$ (Cyc 2-10), and cycles 61-70 (Cyc 60-70). Force readings from pressure transducers changes between 10.4 and $16.2 \%$ compared cycle 2 and cycle 70 .
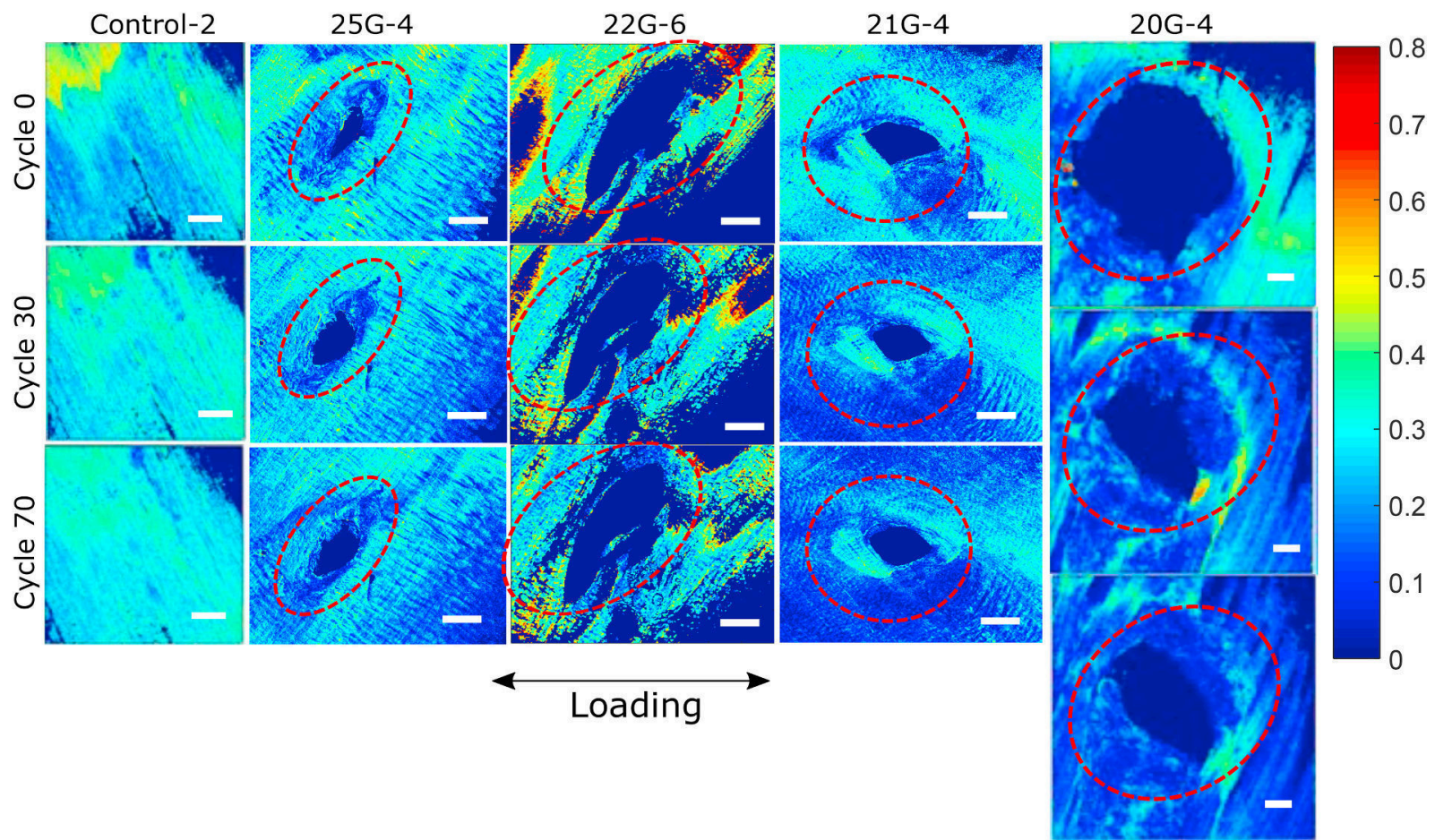

Fig. 4. $I_{2}$ mapping of the collagen fibre from the polarization SHG images. One representative sample was taken from each group (the same samples shown in Fig. 3). In each sample images, the top row (cycle 0 ) images were taken right after the puncture before the cyclic loadings (cycle 0 ), the middle row were images taken after 30 cyclic loadings (cycle 30), and the bottom row were taken after 70 cyclic loadings (cycle 70). All scale bars represent $200 \mu \mathrm{m}$. 
(a) Control - 2

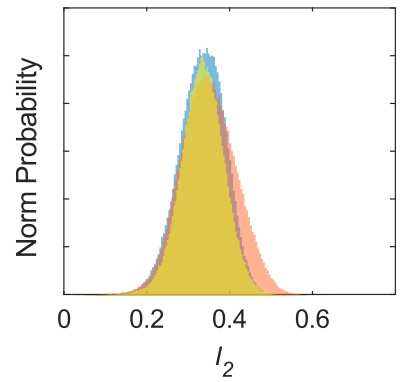

(d) 21G - 4

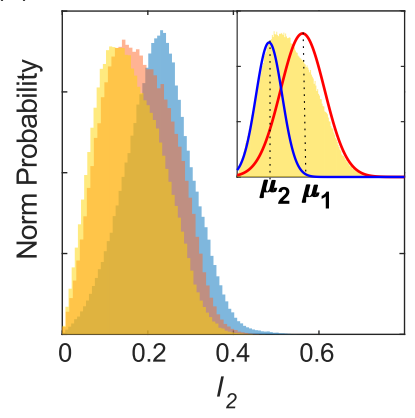

(b) 25G - 4

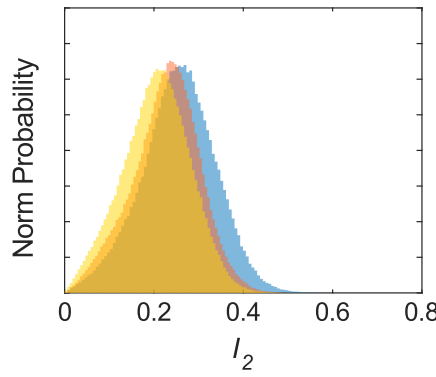

(e) 20G - 4

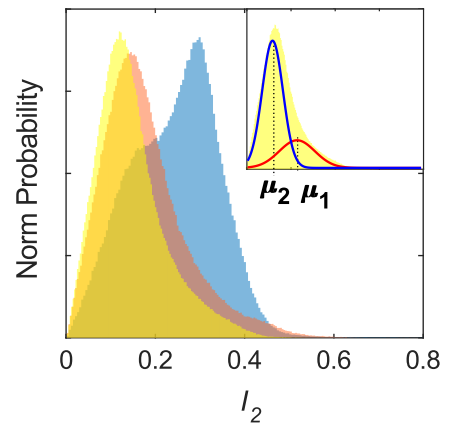

(c) $22 \mathrm{G}-6$

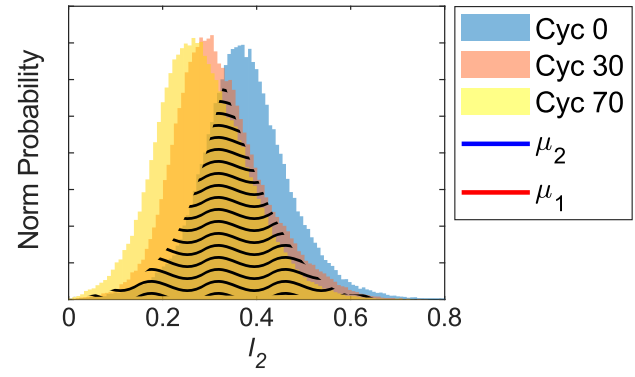

(f)

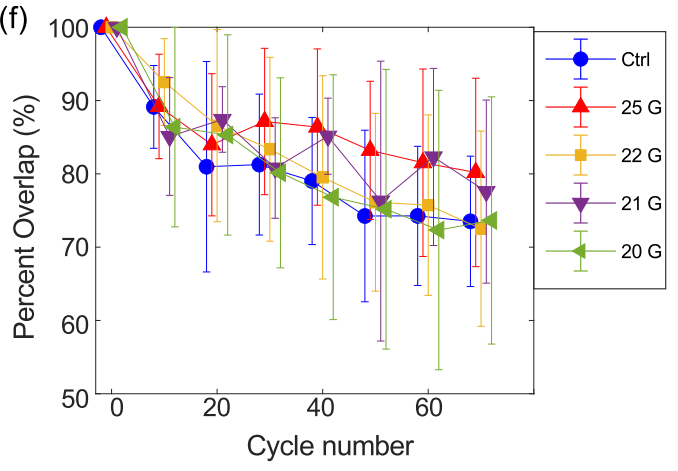

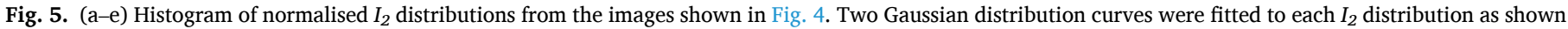

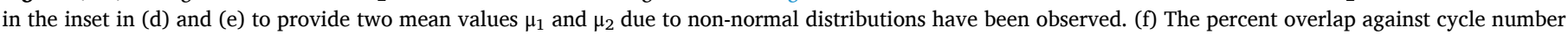

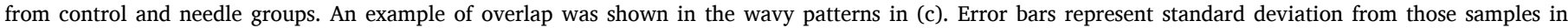
each group.
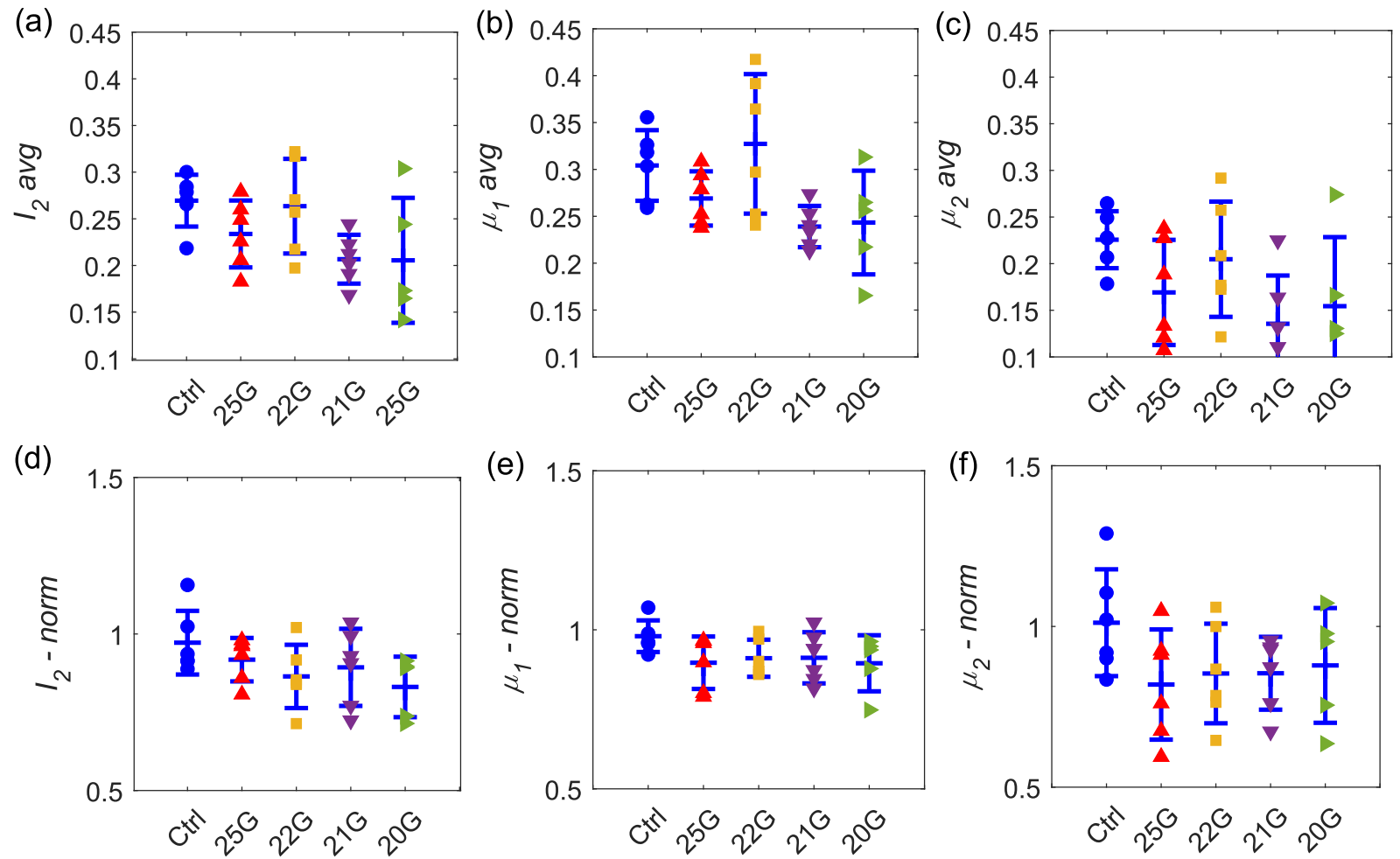

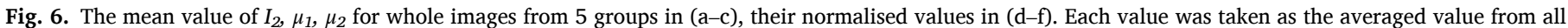
those samples over 70 cycles. Error bars represent the standard deviation from eight samples in each group.

group 22G. The same occurred in $\mu_{1}, \mu_{2}$ values when the distribution was split by Gaussian fitting into two more or less organised populations. For normalised results, decreasing trends were also observed with less variations in $I_{2}$ and $\mu_{1}$ compared to non-normalised values.
Comparing these five needle groups, statistical Kruskal-Wallis tests ( $5 \%$ confidence) showed that there were no significant differences between these groups in non-normalised $I_{2}$ and $\mu_{2}$ where the $p$-values were 0.058 and 0.0596 , while differences could occur in $\mu_{1}$ where $p$-value was 
0.031 . For normalised results, $p$-values were found much higher than $5 \%$ where $p=0.215,0.331,0.134$ for $I_{2}, \mu_{1}, \mu_{2}$, respectively. This indicated no significant differences could be concluded in normalised values between different needle groups. Decreasing trends were observed in those three values against the needle sizes, the correlation coefficients for nonnormalised results against needle sizes $(0.5-0.9 \mathrm{~mm})$ were $-0.744\left(I_{2}\right)$, $-0.512\left(\mu_{1}\right)$, and $-0.810\left(\mu_{2}\right)$; for normalised values the correlation coefficients were $-0.903\left(I_{2}\right),-0.876\left(\mu_{1}\right)$ and $-0.655\left(\mu_{2}\right)$. These indicated that normalised $I_{2}$ and $\mu_{1}$ showed strong linear correlations with needle size.

Two mixing proportions $P_{1}$ and $P_{2}$ of those two populations $\mu_{1}$ and $\mu_{2}$ showed less obvious trend with increasing cycle numbers or needle sizes (Supported Material 2). No significant differences were found among groups (with $5 \%$ confidence) where $p$-values from Kruskal-Wallis tests were 0.380 (non-normalised $P_{1}$ ), 0.423 (non-normalised $P_{2}$ ), 0.292 (normalised $P_{1}$ ) and 0.431 (normalised $P_{2}$ ). Weak correlations were also found where Pearson's correlation coefficients between the needle size and non-normalised $P_{1}$ was 0.579 , between non-normalised $P_{2}$ was -0.593 , between normalised $P_{1}$ was -0.2879 , and between normalised $P_{2}$ was -0.416 . The correlations between cycle numbers varied among needle groups. For non-normalised $P_{1}$, the correlation coefficients between cycle numbers were -0.299 (Control), 0.858 (25G), 0.1263 (22G), $-0.336(21 \mathrm{G})$ and $0.397(20 \mathrm{G})$; for non-normalised $P_{2}$ were 0.291 (Control), -0.863 (25G), 0.051 (22G), 0.285 (21G), and -0.403 (20G). For normalised $P_{1}$, the correlation coefficients between cycle numbers were -0.659 (Control), 0.903 (25G), 0.499 (22G), -0.705 (21G), and $0.758(20 \mathrm{G})$; for normalised $P_{2}$ were -0.052 (Control), -0.809 (25G), 0.768 (22G), 0.440 (21G) and 0.148 (20G).

In order to investigate any progressive damage due to axial cyclic loading, the results of the mean $I_{2}$ values taken from the polarized-SHG images were plotted against cycle number, as shown in Fig. 7 (a). A linear approximation was fitted where the slope was also plotted from one representative sample in each group. The mean slopes of $I_{2}, \mu_{1}, \mu_{2}$ from all the samples separated to each group were plotted in Fig. 7(b-d) and their normalised values were plotted in Fig. 7(e-g). The correlation coefficients between non-normalised $I_{2}$ and cycle numbers were -0.627 (control), -0.893 (25G), -0.851 (22G), -0.978 (21G), -0.924 (20G); and were -0.562 (control), -0.871 (25G), -0.518 (22G), -0.974 (21G) and $-0.924(20 \mathrm{G})$ for normalised $I_{2}$. The correlation coefficients between non-normalised $\mu_{1}$ and cycle numbers were -0.588 (control),
-0.829 (25G), $-0.518(22 \mathrm{G}),-0.937$ (21G), -0.939 (20G); and were -0.582 (control), -0.835 (25G), -0.644 (22G), -0.936 (21G) and -0.934 (20G) for normalised $\mu_{1}$. The correlation coefficients between non-normalised $\mu_{2}$ and cycle numbers were -0.055 (control), -0.962 (25G), -0.955 (22G), -0.925 (21G), -0.798 (20G); and were -0.039 (control), -0.952 (25G), -0.935 (22G), -0.913 (21G) and -0.934 (20G) for normalised $\mu_{2}$. For both normalised and non-normalised results, $I_{2}, \mu_{1}, \mu_{2}$ were less negatively correlated to cycle numbers for the control group compared to the needle groups, which indicated the cyclic loadings had less damaging effects on the control group compared to the needle groups.

Overall decreases were observed in both normalised and unnormalised mean $I_{2}$ slopes with increasing needle size where the correlation coefficient was -0.664 for non-normalised slopes and -0.603 for normalised slopes. Similar trends of decrease also occurred in nonnormalised $\mu_{1}, \mu_{2}$ where correlation coefficients were -0.257 and -0.591 ; however, the decreasing trends were less obvious in normalised slopes where the correlation coefficient was -0.075 for normalised $\mu_{1}$ slope and -0.005 between normalised $\mu_{2}$ slope and needle size. For both normalised and un-normliased slopes, the $p$-values from Kruskal-Wallis tests were also found much higher than $5 \%$ confidence, where $p=$ $0.372,0.228,0.610$ (for un-normliased $I_{2}, \mu_{1}, \mu_{2}$ slopes, respectively) and $p=0.713,0.265,0.662$ (for normalised $I_{2}, \mu_{1}, \mu_{2}$ slopes respectively). These indicated no significant difference was found between groups at 5\% significance level.

\subsection{Cell nuclei images}

AF collagen fibre bundles and cell nuclei were imaged using SHG and TPF microscopy as shown in Fig. 8(a-c) where images were taken from a representative disc sample punctured by a $25 \mathrm{G}$ hypodermic needle at the site of the puncture. Images from a control-group sample are shown in Fig. 8(d-e). In Fig. 8(a) the needle puncture is located at the centre of the image where some rounding in cell shape was found surrounding the puncture in Fig. 8(b) compared to the elongated spindle-shaped cells embedded in unpunctured collagen bundle in Fig. 8(d). Higher magnification of the image in Fig. 8(c) was taken at the corner of the puncture within a square in Fig. 8(b).

This shows the cell nuclei are more irregular, rounder and accumulated near the damage site where the collagen fibre was pushed down
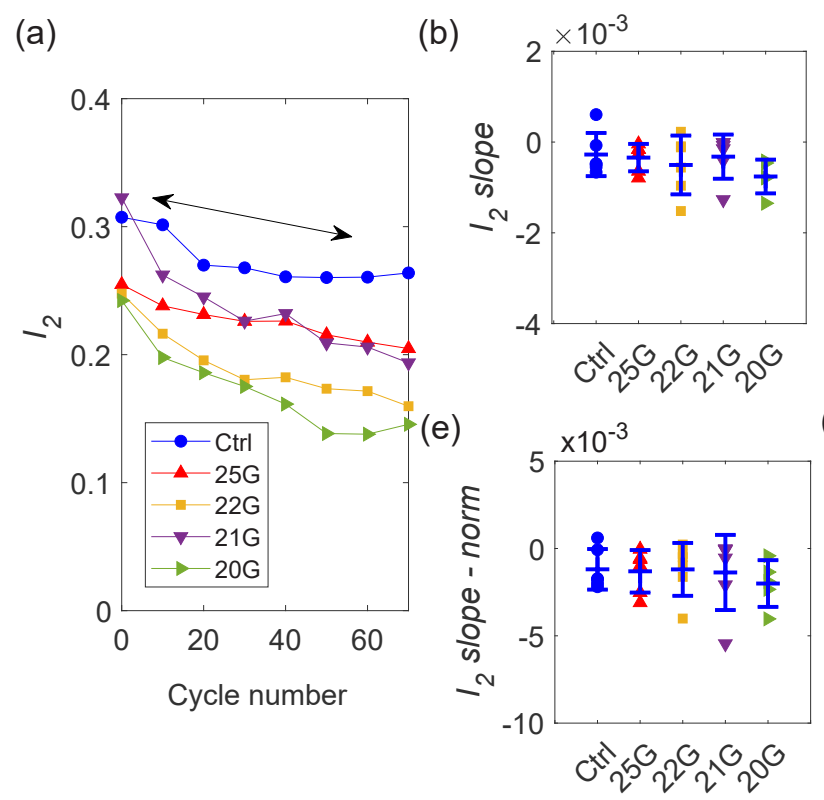

(c)

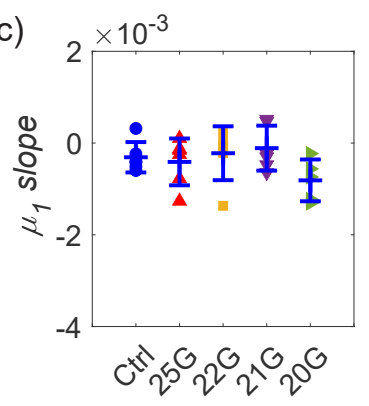

(f)

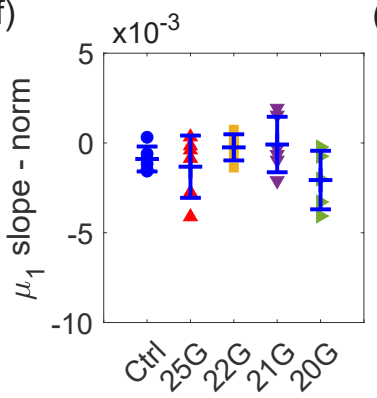

(d)

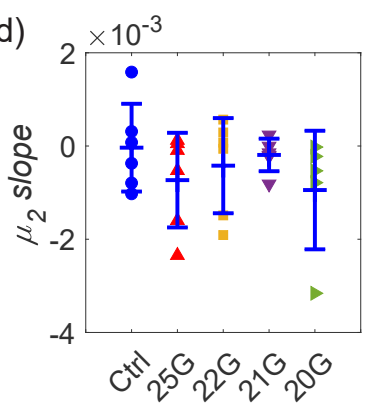

(g)

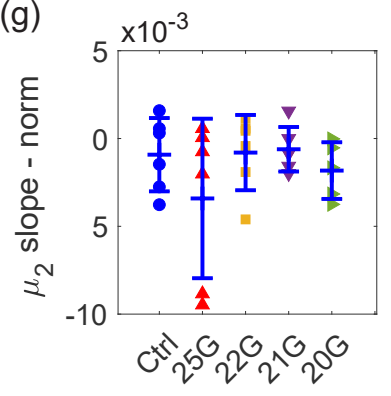

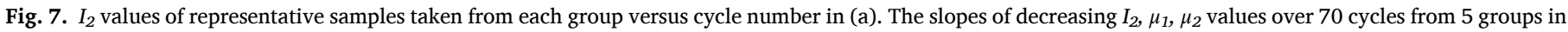

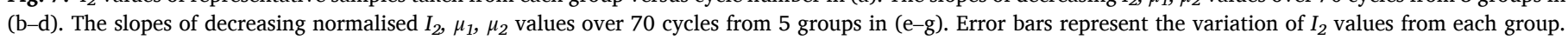


(a)

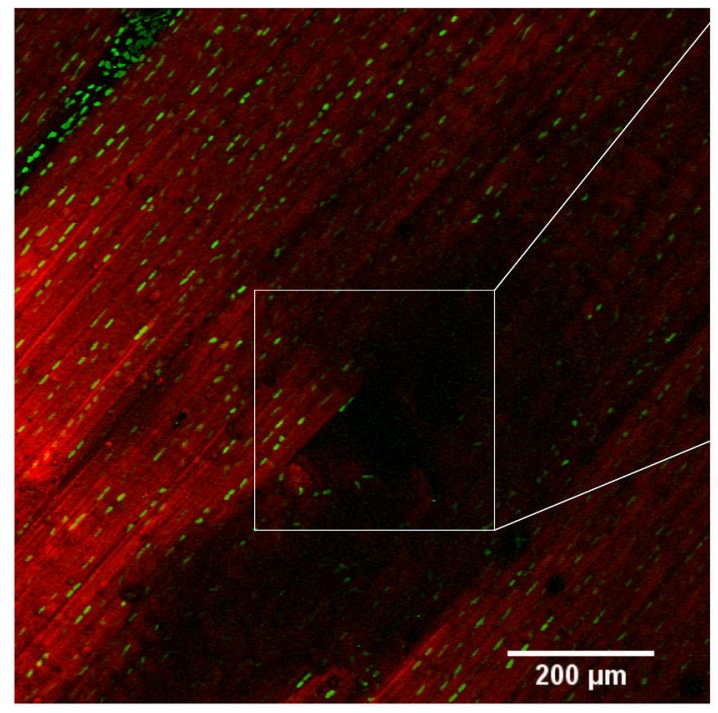

(b)

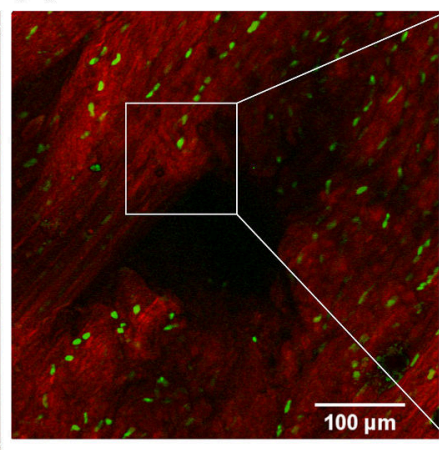

(d)

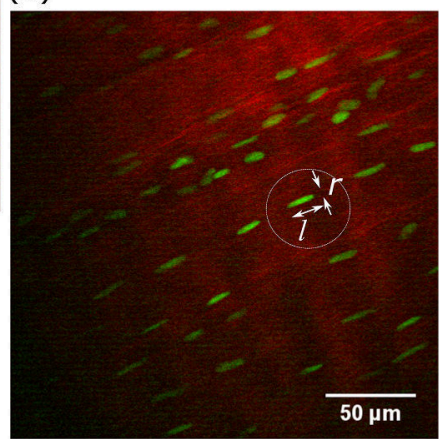

(c)

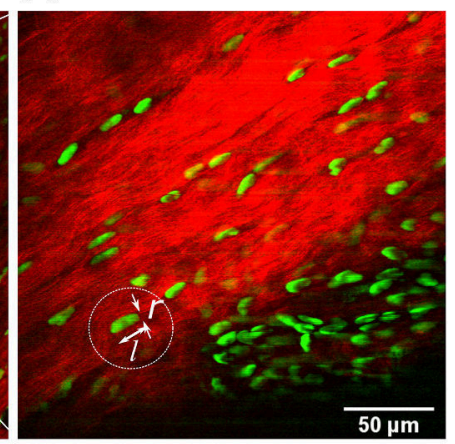

(e)

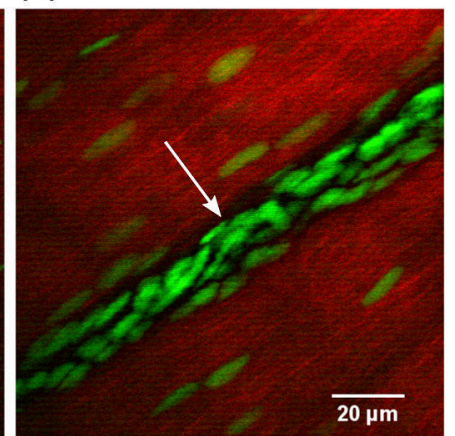

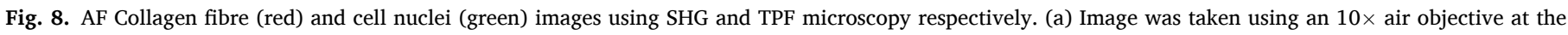

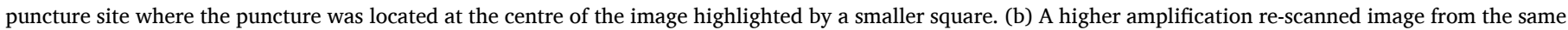

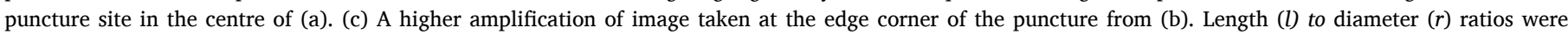

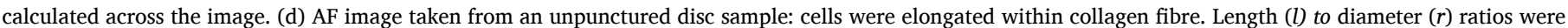

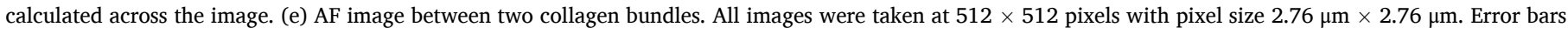
represent (a) $200 \mu \mathrm{m}$, (b) $100 \mu \mathrm{m}$, (c) $50 \mu \mathrm{m}$, (d) $50 \mu \mathrm{m}$ and (e) $20 \mu \mathrm{m}$.

into the disc. Fig. 8(d) was taken from a control sample where cells are thinner and elongated between collagen fibres. Accumulated cells with irregular shapes were also observed between collagen bundles as indicated by small an arrow in Fig. 8(a) and (e). Near the puncture site in Fig. 8(c), the average value of cell length $(l)$ to diameter $(r)$ ratio $(l / r)$ was $2.98 \pm 1.05$ ( 65 cells) which indicated rounder cell shape, compared to cells in unpuncture sample shown in Fig. 8(d) where the cell shape are more elongated with averaged $l$ to $r$ ratio $(l / r) 4.47 \pm 1.29$ (53 cells).

\section{Discussion}

This study aimed to investigate the effect of needle puncture on the outer surface of the annulus fibrosus using SHG. It has previously been found, in animal models, that short-term damage can be effectively restrained when small needles are used, due to the laminated and fibrous structure of the annulus fibrosus (Iatridis et al., 2013). Four different sizes of needles were thus included in this study to investigate the damage to the outer annulus fibrosus. Needle punctures have been suspected to cause long term back pain due to structural damage which can lead to biochemical dysfunction (Michalet et al., 2010), cyclic loadings were applied axially in this study to investigate progressive damage surrounding the puncture. Low-frequency cyclic loading was used in this study since it has previously been found to affect delamination and wall distortion in the annulus (Schollum et al., 2018).We had previously studied the damage further away from the puncture site and the results showed that the needle puncture did not cause any changes in the elastic modulus of annulus fibrosus (Vergari et al., 2017a). However, the tests were performed on annulus fibrosus samples which had been cut into strips. In this study, whole motion segments were thus used with disc samples remaining attached to two vertebrae.

Whilst the amount of dehydration was minimised in the experiments by applying petroleum jelly around the AF and endplates, leaving a small window exposed to the air at the puncture site and by rinsing the puncture site with PBS just before puncture, some sample dehydration will have taken place. Whole motion segments were used to give an as realistic as possible puncture and loading protocol, however these could not be maintained in a water bath, and therefore some water may have been lost from the cut ends of the bone. Additionally an air objective, instead of water or oil objective, was used for the imaging to allow a large enough field of view to cover the whole puncture site, however in doing so moisture could also be lost at the image site. Any dehydration taking place may account for the $-10.2 \%$ of axial strength loss on average within imaging period $2.5 \mathrm{~h}$ on unpunctured. For punctured samples, the axial strength loss was found between $-14.4 \%$ and $-18.9 \%$ on average within imaging period of $3.5 \mathrm{~h}$ while the on AF imaging area was rinsed in between by PBS just before the puncture. Dehydration could also cause minor bone shrinkage and slippage with reduced diameter (Lievers et al., 2007, 2010), while the main dehydration in this study was still assumed to come from the direct imaging area exposed to the air as the dehydration from the vertebrae would require the water to diffuse through the endplates. Needle damage would also cause loss of axial strength in the needle groups (Michalek et al., 2010; Michalek and Iatridis, 2012; Zirbel et al., 2013). In this study, although not being able to differentiate two factors explicitly from the same samples, axial strength loss in the needle group were $4.2-8.7 \%$ greater compared to the control group which could come from the needle puncture.

Due to the geometry of the microscope, to carry out images of the tissue deformation whilst the disc was being punctured was not possible which is one of the main limitations of SHG. It was thus not possible to make any direct comparison before and after the puncture on the same sample surface as it was inevitable to puncture at a different spot. This was also due to the small needle diameters $(0.5-0.9 \mathrm{~mm})$ compared to 
the size of $5 \times 5 \mathrm{~mm}^{2}$ area left for imaging. Otherwise to make direct comparision between the collagen fibre orientations at the site before and after puncture would have further enhanced the study and provided valuable information about how the size and shape of the puncture and the amount of peripheral damage were affected. The SHG imaging was from a single focal plane with a depth of approximately $7 \mu \mathrm{m}$, and due to the scattering nature of AF tissue we were only able to image close to the sample surface. Changes out of the focal plane caused by the needle puncture and loading therefore could not be monitored. In addition, the circumferential locations of punctures were not recorded in this study. The main challenge also came from the fact that the imaging was limited to the $7 \mu \mathrm{m}$ focal depth and the imaging windows could not be all located at posterior portion after the initial dissection. The microstructure of AF can vary on the same disc where outer AF were found thinner and the fibres in the adjacent layers were found to be more in parallel in posterior portion compared to anterior or lateral portion (Markolf and Morris, 1974). Clinical lumbar puntures locate at posterior part of the disc which are expected to cause disc damage more easily. To determine long-term damage in terms of puncture locations would require larger sample sizes and precise microdissections while this study presented a preliminary results of prepherial damages on outer AF surface after short period of cyclic loadings.

Two different types of needles, the hypodermic and spinal needle, with two different sizes were used in this study. The damage caused by the needle shape were not compared in this study. Some of the collagen bundles were cut through with part of the collagen fibres being pushed inside or lifted up (Fig. 2). Those collagen fibres which were lifted up were out of the imaging focal depth $(7 \mu \mathrm{m})$ and had low SHG intensity and thus were not included in this study. In Fig. 3, the puncture size change could indicate the loading might have caused tearing in the puncture in which less shrinkage occurred in the direction perpendicular to the loading and larger needles caused more tearing with negative correlation to needle size. However, the complex combination of mechanical stretching, orientation of needle tip openings, the cutting of the collagen fibres, drying of the sample surface could also take place. Similar to the clinical applications, the openings of the tips were random and not monitored in this study. The effect of dehydration could also contribute to shape changes which is the limitation in this study. Larger sample sizes were required to verify the significant changes under hydrated states. Furthermore, all the samples were fixed on the rig, those changes (shrinkage or expansion) have been found occurred only locally, i.e. within adjacent fibre bundles, while this study only focused on the imaging site of around $1.5 \mathrm{~mm} \times 1.5 \mathrm{~mm}$ area with pixel size 2 $\mu \mathrm{m} \times 2 \mu \mathrm{m}$ to cover the whole puncture site and compare the progressive damages.

From the polarized-SHG imaging results, the overall $I_{2}$ value decreases with increasing needle size, as shown in Fig. 6. Non-normal distributions were found in $I_{2}$ and two Gaussian curves were fitted with two mean values $\left(\mu_{1}, \mu_{2}\right)$ being compared. Those two values represent the populations of more organised $\left(\mu_{1}\right)$ and less organised $\left(\mu_{2}\right)$ collagen molecules across the whole images. However, how those two populations relate to damages at molecular level such as damages on different molecules and thus collagen network will require further invests with larger sample sizes and higher magnification objectives imaging the damaged fibrils. Each fitted Gassian distribution curve was described in three parameters, the mean $(\mu)$, the mixing proportion $(P)$ and the standard deviation $(\sigma) .26$ out of 29 samples having decreasing $\mu_{1}$ with increasing cycle number indicated an overall shift of average values in more organised collagen population. However, no obvious trends were observed in $P$ and $\sigma$ which suggested more complex mechanism were taken places where two polulations did not simply shift rather than changing in both proportions and distributions.

A progressive decrease in overall $I_{2}$ over short-term cyclic loading was observed, with a larger needle size having more decrease as shown in Fig. 7. Strong correlations were found between cycle numbers and $I_{2}$, $\mu_{1}$ and $\mu_{2}$ in needle groups with correlation coefficients higher than 0.79 , while weaker correlations were found in control group with correlation coefficients less than 0.59 . These indicated the needle damage could cause more progressive damage. It can be further explored and applied in clinical practice, since this result suggests that using larger needle can cause more long-term damage. However, the statistical results in this study, although the percent overlap $I_{2}$ distribution decreased with increasing cyclic loadings for all samples, no statistical differences could be concluded between needle groups. Similarly, decreasing trends were found in normalised and non-normalised $I_{2}, \mu_{1}, \mu_{2}$ and their slopes, while no significant difference could be concluded within $5 \%$ confidence. These also verify the results from other research groups (Iatridis and Commentary, 2012; Cuellar et al., 2016) in which mild or moderate damage have been observed. While it does not come as a surprise that using a larger needle can increase damage, this study showed that the reason for this increase is not the larger hole produced by the larger needle, but rather the microscopic local disruption around it.

Finally, the outer AF cell nuclei at the puncture site showed rounded shape (Fig. 8). Clustered and round cells have also been found surrounding puncture and between bundles, which has been little studied. Round cells are often observed in aging and degenerative discs, instead of elongated fibroblast-like in healthy young discs (Johnson and Roberts, 2003; Pattappa et al., 2012; Gruber and Hanley, 2000; Gruber et al., 2003). Cell shape can influence cell function and mitogenesis capacity, and in turn the interactions between cells and extracellular matrix can regulate cell shape, cell gene expression and cell functions (Grad et al., 2010; Bruehlmann et al., 2002). In this study, disc samples had undergone the freezing and thawing processes, cell nuclei rounding could be due to membrance lysis during the process, or the needle damages of the extracellular matrix causing the AF cells return to their lower mechanical energy state which tend to be round in nature. To have clearer understanding of the cause would require cell membrane of cytoskeletal imaging which will be left in future work.

In this study, the number of samples was small with only 5 to 6 samples in each group and some results have not shown statistical differences in this study, further improvement including the effect of cyclic loadings with longer period would require larger sample sizes with samples kept in hydrated state for statistical studies.

\section{Conclusion}

In this study the progressive damages on collagen fibres in outer annulus fibrosus of bovine intervertebral discs caused by needle puncture were investigated using polarization-resolved SHG at the site of puncture. By using a generic model to analyse the SHG response, the degree of the collagen fibril organization was presented pixel by pixel. There were two main categories of the puncture shape which agree with the results from other research groups, corresponding to more or less damage induced on the collagen organization. Larger needles have been found to cause more moderate damages over short-term cyclic loadings while statistically no significant differences can be concluded with such small sample sizes. There are a few limitations in this study, including the dehydration which caused $10-19 \%$ of axial strength loss, the SHG limited to prepheral images, and small sample size.

In conclusion, although with small sample sizes, mechanical effects of needle puncture found in this study on disc microstructure were not significantly affected by needle type, and only marginally by needle size. This confirms that puncturing the disc for diagnostic or therapeutic aims should be avoided when possible, because all types and size of needles induced similar damage, and it should be done with smaller needle size when necessary.

\section{Author statement}

Jau-Yi Wang: Conceptualization, Methodology, Data Curation, Software, Investigation, Resources, Formal Analysis, Writing - original draft, Visualization, Validation. 
Jessica C Mansfield: Conceptualization, Methodology, Data Curation, Software, Resources, Writing - Review\& Editing.

Sophie Brasselet: Conceptualization, Methodology, Data Curation, Software, Formal Analysis, Writing - Review \& Editing.

Claudio Vergari: Conceptualization, Methodology, Data Curation, Software, Resources, Writing - Review\& Editing.

Judith R Meakin: Conceptualization, Writing - Review\& Editing, Supervision, Project administration, Funding acquisition.

C Peter Winlove: Conceptualization, Writing - Review\& Editing, Supervision, Project administration, Funding acquisition.

\section{Declaration of competing interest}

The authors declare that they have no known competing financial interests or personal relationships that could have appeared to influence the work reported in this paper.

\section{Acknowledgements}

The authors thank the Henry Smith Charity for funding this study [grant number 20141464].

\section{Appendix A. Supplementary data}

Supplementary data to this article can be found online at https://doi. org/10.1016/j.jmbbm.2021.104458.

\section{References}

Anceau, C., Brasselet, S., Zyss, J., 2005. Local orientational distribution of molecular monolayers probed by nonlinear microscopy. Chem. Phys. Lett. 411, 98-102. https://doi.org/10.1016/j.cplett.2005.06.018.

Ávila, F.J., del Barco, O., Bueno, J.M., 2015. Polarization dependence of aligned collagen tissues imaged with second harmonic generation microscopy. J. Biomecial Optics 20, 086001. https://doi.org/10.1117/1.JBO.20.8.086001.

Aït-Belkacem, D., Roche, M., Duboisset, J., Ferrand, P., Brasselet, S., Guilbert, M. Sockalingum, G.D., Jeannesson, P., 2012. Microscopic structural study of collagen aging in isolated fibrils using polarized second harmonic generation. J. Biomed. Opt. 17, 080506 https://doi.org/10.1117/1.JBO.17.8.8080506.

Bernard, T.N., 1990. Lumbar discography followed by computed tomography. Spine 15 609-707. http://europepmc.org/abstract/MED/2145643.

Bowles, R.D., Williams, R.M., Zipfel, W.R., Bonassar, L.J., 2010. Self-assembly of aligned tissue-engineered annulus fibrosus and intervertebral disc composite via collagen gel contraction. Tissue Eng. 16, 1339-1348. https://doi.org/10.1089/ten. tea.2009.0442.

Brasselet, S., 2011. Polarization-resolved nonlinear microscopy: application to structural molecular and biological imaging. Adv. Opt Photon 3, 205-271. https://doi.org/ 10.1364/AOP.3.000205.

Brown, S., Rodrigues, S., Sharp, C., Wade, K., Broom, N., McCakk, I.W., Roberts, S., 2016. Staying connected: structural integration at the intervertebral disc-vertebra interface of human lumbar spines. Eur. Spine J. 26, 248-258. https://doi.org/10.1007/ s00586-016-4560-y.

Bruehlmann, S.B., Rattner, J.B., Matyas, J.R., Duncan, N.A., 2002. Regional variations in the cellular matrix of the annulus fibrosus of the intervertebral disc. J. Anat. 201, 159-171. https://doi.org/10.1046/j.1469-7580.2002.00080.x.

Bueno, J.M., Ávila, F.J., Artal, P., 2016. Second harmonic generation microscopy: a too for quantitative analysis of tissues. Microsc. Anal.: Chap 5. https://doi.org/10.5772 63493. Stefan G. Stanciu, IntechOpen.

Buys, T.S., De Clerk, K., 1972. Bi-Gaussian fitting of skewed peaks. Anal. Chem. 44, 1273-1275. https://doi.org/10.1021/ac60315a005.

Campagnola, P., 2011. Second harmonic generation imaging microscopy: applications to diseases diagnostics. Anal. Chem. 83, 3224-3231. https://doi.org/10.1021/ ac1032325.

Carragee, E.J., Lincoln, T., Parmar, V.S., Alamin, T., 2006. A gold standard evaluation of the "discogenic pain" diagnosis as determined by provocative discography. Spine 31 2115-2123. https://doi.org/10.1097/01.brs.0000231436.30262.dd.

Chen, X., Nadiarynkh, O., Plotnikov, S., Campagnola, P.J., 2012. Second harmonic generation microscopy for quantitative analysis of collagen fibrillar structure. Nat. Protoc. 7, 654-669. https://doi.org/10.1038/nprot.2012.009.

Cohen, S.P., Williams, S., Kurihara, C., Griffith, S., Larkin, T.M., 2005. Nucleoplasty with or without intradiscal electrothermal therapy (IDET) as a treatment for lumbar herniated disc. Spinal Discard Tech 18, S119-S123. https://doi.org/10.1097/01. bsd.0000127823.54485.3f.

Cox, G., Kable, E., 2006. Second-harmonic imaging of collagen. Methods Mol. Biol. 319, 15-35. https://doi.org/10.1007/978-1-59259-993-6 2.

Cuellar, J.M., Stauff, M.P., Herzog, R.J., Carrino, J.A., Baker, G.A., Carragee, E.J., 2016. Does provocative discography cause clinically important injury to the lumbar intervertebral disc? A 10-year matched cohort study. Spine J. 16, 273-280. https:// doi.org/10.1016/j.spinee.2015.06.051.

Deniset-Besseau, A., Duboisset, J., Benichou, E., Hache, F., Brevet, P.-F., SchanneKlein, M.-C., 2009. Measurement of the second-order hyperpolarizability of the collagen triple helix and determination of its physical origin. J. Phys. Chem. B 113, 437-445. https://doi.org/10.1021/jp9046837.

Dittmar, R., 2008. Intervertebral Disc Visualization by Combined Two-Photon Excitation Fluorescence and Second-Harmonic Generation Microscopy (Master Dissertation). Retrieved from Eindhoven University of Technology. http://www.mate.tue.nl/mat e/pdfs/8927.pdf.

Duboisset, J., Aït-Belkacem, D., Roche, M., Rigneault, H., Brasselet, S., 2012. Generic model of the molecular orientational distribution probed by polarization-resolved second-harmonic generation. Phys. Rev. 85, 043829 https://doi.org/10.1103/ PhysRevA.85.043829.

Ehrlich, G.E., 2003. Low back pain. Bull. World Health Organ. 81, 671-676. http s://www.who.int/bulletin/volumes/81/9/Ehrlich.pdf.

Grad, S., Alini, M., Eglin, D., Sakai, D., Mochida, J., Mahor, S., Collin, E., Dash, B., Pandit, A., 2010. Cells and biomaterials for intervertebral disc regeneration. Synthesis lectures on tissue engineering 5, 1-104. https://doi.org/10.2200/ S00250ED1V01Y201006TIS005.

Gruber, H.E., Hanley, E.N., 2000. Human disc cells in monolayer vs 3D culture: cell shape, division and matrix formation. BMC Muscoskel. Disord. 1, 1. https://doi.org/ 10.1186/1471-2474-1-1.

Gruber, H.E., Ingram, J.A., Leslie, K., Norton, H.J., Hanley Jr., E.N., 2003. Cell shape and gene expression in human intervertebral disc cells: in vitro tissue engineering studies. Biotech. Histochem. 78, 109-117. https://doi.org/10.1080/ 10520290310001593793.

Guyer, R.D., Ohnmeiss, D.O., discography, Lumbar, 2003. Spine J. 3, 11S-27S. https ://journals.lww.com/spinejournal/Fulltext/1996/02010/Lumbar_Discography.31. aspx.

Hayes, A.J., Isaacs, M.D., Hughes, C., Caterson, B., Ralphs, J.R., 2011. Collagen fibrillogenesis in the development of the annulus fibrosus of the intervertebral disc. Eur. Cell. Mater. 22, 226-241. https://doi.org/10.22203/eCM.v022a18.

Hickey, D.S., Hukins, D.W., 1981. Collagen fibril diameters and elastic fibres in the annulus fibrosus of human fetal intervertebral disc. J. Anat. 133, 351-357. https:// www.ncbi.nlm.nih.gov/pmc/articles/PMC1167606/pdf/janat00223-0032.pdf.

Hickey, D.S., Hukins, D.W., 1982. Aging changes in the macromolecular organization of the intervertebral disc: an X-ray diffraction and electron microscopic study. Spine 7 (3), 234-242. https://europepmc.org/abstract/med/7112237.

Iatridis, J.C., Commentary, A.C. Hecht, 2012. Does needle injection cause disc degeneration? News in the continuing debate regarding pathophysiology associated with intradiscal injections. Spine J. 12, 336-338. https://doi.org/10.1016/j. spinee.2012.03.006.

Iatridis, J.C., Gwynn, L.A., 2004. Mechanisms for mechanical damage in the intervertebral disc annulus fibrosus. J. Biomech. 37, 1165-1175. https://doi.org/ 10.1016/j.jbiomech.2003.12.026.

Iatridis, J.C., Michalek, A.J., Purmessur, D., Korecki, C.L., 2009. Localized intervertebral disc injury leads to organ level changes in structure, cellularity, and biosynthesis. Cell. Mol. Bioeng. 2, 437-447. https://doi.org/10.1007/s12195-009-0072-8.

Iatridis, J.C., Nicoll, S.B., Michalek, A.J., Walter, B.A., Gupta, M.S., 2013. Role of biomechanics in intervertebral disc degeneration and regenerative therapies: what needs repairing in the disc and what are promising biomaterials for its repair? Spine J. 13, 243-262. https://doi.org/10.1016/j.spinee.2012.12.002.

Izzo, R., Popolizio, T., D’Aprile, P., Muto, M., 2015. Spinal pain. Eur. J. Radiol. 84 746-756. https://doi.org/10.1016/j.ejrad.2015.01.018.

Johnson, W.E., Roberts, S., 2003. Human intervertebral disc cell morphology and cytoskeletal composition: a preliminary study of regional variations in health and disease. J. Anat. 203, 605-612. https://doi.org/10.1046/j.1469-7580.2003.00249. $\mathrm{x}$.

Keorochana, G., Johnson, J.S., Taghavi, C.E., Liao, J.-C., Lee, K.-B., Yoo, J.H., Ngo, S.S., Wang, J.C., 2010. The effect of needle size inducing degeneration in the rat caudal disc: evaluation using radiograph, magnetic resonance imaging, histology, and immunohistochemistry. Spine J. 10, 1014-1023. https://doi.org/10.1016/j. spinee. 2010.08.013.

Klein, J.A., Jukins, D.W., 1982. X-ray diffraction demonstrates reorientation of collagen fibres in the annulus fibrosus during compression of the intervertebral disc. Biochim. Biophys. Acta 717, 61-64. https://doi.org/10.1016/0304-4165(82)90380-4.

Kleinstueck, F.S., Diederich, C.J., Nau, W.H., Puttlitz, C.M., Smith, J.A., Bradford, D.S., Lotz, J.C., 2001. Acute biomechanical and histological intradiscal electrothermal therapy on human lumbar discs. Spine 20, 2198-2207. https://journals.lww.co

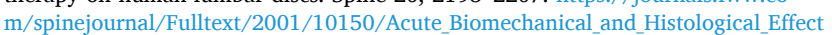
s. of. 9 . aspx.

Kobielarz, M., Szotek, S., Glowacki, M., Dawidowics, J., Pezowicz, C., 2016. Qualitative and quantitative assessment of collagen and elastin in annulus fibrosus of the physiologic and scoliotic intervertebral discs. J Mech. Behav. Biomed. Mater. 62, 45-56. https://doi.org/10.1016/j.jmbbm.2016.04.033.

Korecki, C.L., Costi, J.J., Iatridis, J.C., 2008. Needle puncture injury affects intervertebral disc mechanics and biology in an organ culture model. Spine 33, 235-241. https:// doi.org/10.1097/BRS.0b013e3181624504.

Lacomb, R.I., Nadiarnykh, O., Campagnola, P.J., 2008. Quantitative second harmonic generation imaging of the diseased state osteogenesis imperfecta: experiment and simulation. Biophys. J. 94, 4504-4514. https://doi.org/10.1529/ biophysj.107.114405.

Lievers, W.B., Lee, V., Arsenault, S.M., Waldman, S.D., Pilkey, A.K., 2007. Specimen size effect in the volumetric shrinkage of cancellous bone measured at two levels of 
dehydration. J. Biomech. 40, 1903-1909. https://doi.org/10.1016/j. jbiomech.2006.09.002.

Lievers, W.B., Poljsak, A.S., Waldman, S.D., Pilkey, A.K., 2010. Effects of dehydrationinduced structural and material changes on the apparent modulus of cancellous bone. Med. Eng. Phys. 328, 921-925. https://doi.org/10.1016/j. medengphy.2010.06.001.

Manchikanti, L., Glaser, S.E., Wolfer, L., Derby, R., Cohen, S.P., 2009. Systematic Review of lumbar discography as a diagnostic test for chronic low back pain. Pain Physician 12, 541-559. https://www.painphysicianjournal.com/current/pdf?article=MTIxOA $\% 3 \mathrm{D} \% 3 \mathrm{D} \&$ journal $=49$.

Mansfield, J.C., Winlove, C.P., Moger, J.J., Matcher, S.J., 2008. Collagen fiber arrangement in normal and diseased cartilage studied by polarization sensitive nonlinear microscopy. J. Biomed. Opt. 13 (4), 044020 https://doi.org/10.1117/ 1.2950318 .

Mansfield, J., Yu, J., Attenburrow, D., Moger, J., Tirlapur, U., Urban, J., Cui, Z., Winlove, C.P., 2009. The elastin network: its relationship with collagen and cells in articular cartilage as visualized by multiphoton microscopy. J. Anat. 215, 682-691. https://doi.org/10.1111/j.1469-7580.2009.01149.x.

Mansfield, J.C., Mandalia, V., Toms, A., Winlove, C.P., Brasselet, S., 2019. Collagen reorganization in cartilage under strain probed by polarization sensitive second harmonic generation microscopy. J. R. Soc. Interface 16. https://doi.org/10.1098/ rsif.2018.0611.

Markolf, K.L., Morris, J.M., 1974. The structural components of the intervertebral disc. A study of their contributions to the ability of the disc to withstand compressive forces. J Bone Joint Surg Am. Jun 56 (4), 675-687. https://journals.lww.com/jbjsjournal/Fu 1ltext/1974/56040/The Structural Components of the Intervertebral.3.aspx.

Masuda, K., Aota, Y., Muehleman, C., et al., 2005. A novel rabbit model of mild, reproducible disc degeneration by an annulus needle puncture: correlation between the degree of disc injury and radiological and histological appearances of disc degeneration. Spine 30, 5-14. https://doi.org/10.1097/01. brs.0000148152.04401.20.

Matcher, S.J., 2015. What can biophotonics tell us about the 3D microstructure of articular cartilage? Quant. Imag. Med. Surg. 5, 143-158. https://doi.org/10.3978/j. issn.2223-4292.2014.12.03.

Michalek, A.J., Iatridis, J.C., 2012. Height and torsional stiffness are most sensitive to annular injury in large animal intervertebral discs. Spine J. 12, 425-432. https://doi. org/10.1016/j.spinee.2012.04.001.

Michalek, A.J., Funabashi, K.L., Iatridis, J.C., 2010. Needle puncture injury of the rat intervertebral disc affects torsional and compressive biomechanics differently. Eur. Spine J. 19, 2110-2116. https://doi.org/10.1007/s00586-010-1473-z.

Michalet, A.J., Buckley, M.R., Bonassae, L.J., Cohen, I., Iatridis, J.C., 2010. The effects of needle puncture injury on microscale shear strain in the intervertebral disc anulus fibrosus. Spine J. 10, 1098-1105. https://doi.org/10.1016/j.spinee.2010.09.015.

Nosikova, Y.S., Santerre, J.P., Grynpas, M., Gibson, G., Kandel, R.A., 2012. Characterization of the annulus fibrosus-vertebral body interface: identification of new structural features. J. Anat. 221, 577-589. https://doi.org/10.1111/j.14697580.2012.01537.x.

Pattappa, G., Li, Z., Peroglio, M., Wismer, N., Alini, M., Grad, S., 2012. Diversity of intervertebral disc cells: phenotype and function. J. Anat. 221, 480-496. https://doi. $\operatorname{org} / 10.1111 /$ j.1469-7580.2012.01521.x.

Pezowicz, C.A., Robertson, P.A., Broom, N.D., 2005. Intralamellar relationships within the collagenous architecture of the annulus fibrosus imaged in its fully hydrated state. J. Anat. 207, 299-312. https://doi.org/10.1111/j.1469-7580.2005.00467.x.
Pollintine, P., Findlay, G., Adams, M.A., 2005. Intradiscal electrothermal therapy can alter compressive stress distributions inside degenerated intervertebral discs. Spine 30, E134-E139. https://doi.org/10.1097/01.brs.0000155559.24555.fc.

Reed, D.A., Yotsuya, M., Gubareva, P., Toth, P.T., Bertagna, A., 2019. Two-photon fluorescence and second harmonic generation characterization of extracellular matrix remodeling in post-injury murine temporomandibular joint osteoarthritis. PloS One 14, e0214072. https://doi.org/10.1371/journal.pone.0214072.

Reiser, K.M., Bratton, C., Yankelevich, D.R., Knoesen, A., Rocha-Mendoza, I., Lotz, J., 2007. Quantitative analysis of structural disorder in intervertebral disks using second harmonic generation imaging: comparison with morphometric analysis. J. Biomed. Opt. 12, 064019 https://doi.org/10.1117/1.2812631.

Schollum, M.L., Robertson, P.A., Broom, N.D., 2007. A microstructural investigation of intervertebral disc lamellar connectivity: detailed analysis of the translamellar bridges. J. Anat. 214, 805-816. https://doi.org/10.1111/j.1469-7580.2009.01076.

Schollum, M.L., Wade, K.R., Robertson, P.A., Thambyah, A., Broom, N.D., 2018. A microstructural investigation of disc disruption induced by low frequency cyclic loading. Spine 43, E132-E142. https://doi.org/10.1097/BRS.0000000000002278.

Scott, J.E., 1988. Proteoglycan-fibrillar collagen interactions. Biochem. J. 252, 313-323. https://doi.org/10.1042/bj2520313.

Sobajima, S., Kompel, J.F., Kim, J.S., et al., 2005. A slowly progressive and reproducible animal model of intervertebral disc degeneration characterized by MRI, X-ray, and histology. Spine 30, 15-24. https://doi.org/10.1097/01.brs.0000148048.15348.9b.

Tavakoli, J., Elliott, D.M., Costi, J.J., 2017. The ultra-structure organization of the elastic network in the intra- and inter-lammellar matric of the intervertebral disc. Acta Biomater. 58, 269-277. https://doi.org/10.1016/j.actbio.2017.05.036.

Tilbury, K., Lien, C.-H., Chen, S.-J., Campagnola, P.J., 2014. Differentiation of Col I and $\mathrm{Col}$ III isoforms in stromal models of ovarian cancer by analysis of second harmonic generation polarization and emission directionality. Biophys. J. 106, 354-365. https://doi.org/10.1016/j.bpj.2013.10.044.

Tsou, H.-S., Chao, S.-C., Kao, T.H., Yin, J.-J., Hsu, H.-C., Shen, C.-C., Chen, H.-T., 2010. Intradiscal electrothermal therapy in the treatment of chronic low back pain: experience with 93 patients. Surg. Neurol. 1, 37-51. https://doi.org/10.4103/21527806.67107.

Vergari, C., Mansfield, J.C., Meakin, J.R., Winlove, C.P., 2016. Lamellar and fibre bundle mechanics of the annulus fibrosus in bovine intervertebral disc. Acta Biomater. 37, 14-20. https://doi.org/10.1016/j.actbio.2016.04.002.

Vergari, C., Mansfield, J., Chan, D., Clarke, A., Meakin, J.R., Winlove, C.P., 2017a. The effects of needle damage on annulus fibrosus micromechanics. Acta Biomater. 63, 274-282. https://doi.org/10.1016/j.actbio.2017.09.015.

Vergari, C., Chan, D., Clarke, A., Mansfield, J.C., Meakin, J.R., Winlove, C.P., 2017b. Bovine and degenerated human annulus fibrosus: a microstructural and micromechanical comparison. Biomech. Model. Mechanobiol. 16, 1475-1484. https://doi.org/10.1007/s10237-017-0900-z.

Vergroesen, P.-P.A., Kingma, I., Emanuel, K.S., Hoogendoorn, R.J.W., Welting, T.J., van Royen, B.J., van Dieën, J.H., Smit, T.H., 2015. Mechanics and biology in intervertebral disc degeneration: a vicious circle. Osteoarthritis Cartilage 23, 1057-1070. https://doi.org/10.1016/j.joca.2015.03.028.

Yentis, S.M., Hirsch, N.P., Ip, J., 2013. Anaesthesia and Intensive Care A-Z E-Book: an Encyclopedia of Principles and Practice. FRCA Study Guides, $5^{\text {ed. }}$ Elsevier Health Sciences, ISBN 9780702053757.

Zirbel, S.A., Stolworthy, D.K., Howell, L.L., Bowden, A.E., 2013. Intervertebral disc degeneration alters lumbar spine segmental stiffness in all modes of loading under a compressive follower load. Spine J. 13, 1134-1147. https://doi.org/10.1016/j. spinee.2013.02.010. 